\title{
VOLATILITY ESTIMATION ON THE BASIS OF PRICE INTENSITIES
}

\author{
FRANK GERHARD AND NIKOLAUS HAUTSCH \\ FRANK.GERHARD@UNI-KONSTANZ.DE NIKOLAUS.HAUTSCH@UNI-KONSTANZ.DE \\ CENTER OF FINANCE AND ECONOMETRICS (COFE) \\ UNIVERSITY OF KONSTANZ
}

\begin{abstract}
This paper investigates the use of price intensities to estimate volatilities based on high-frequency data. We interpret the conditional probability for the occurrence of a price event within a certain time horizon as a risk measure which allows us to obtain an estimator of the conditional volatility per time. This kind of volatility estimation solves the problem of an appropriate aggregation level by defining explicitly price events. To consider grouping caused by the nontrading period overnight we use a categorical duration model. This model allows us to take into account that durations which occur overnight can only be registered by a lower and an upper bound. The use of price durations based on different tick sizes makes it possible to investigate volatility patterns depending on different aggregation levels. Seasonalities are taken into account by including regressors based on a flexible Fourier form based on intraday and time to maturity seasonalities. Testing for serial correlation and controlling for unobservable heterogeneity permits us to check for misspecification on different aggregation levels. Empirical results are based on intraday transaction data of Bund Future trading at the LIFFE in London.
\end{abstract}

October 20, 1999.

JEL Classifications: C25 C41 G14 G15.

Keywords: Volatility estimation, price intensity, high-frequency data, grouped proportional hazard model, intraday and time to maturity seasonalities.

For valuable comments we would like to thank Joachim Grammig, Dieter Hess, Joachim Inkmann, Stefan Klotz, Michael Lechner, Winfried Pohlmeier and Marno Verbeek. We are also grateful to participants of the 26th meeting of the European Finance Association in Helsinki and workshop participants at the University of Konstanz. The authors would like to thank the Center of Finance and Econometrics (CoFE) for financial support. All remaining errors are our sole responsibility. 


\section{INTRODUCTION}

The road to a feasible estimation of risk measures based on intraday data is strewed with considerable problems. Some of them originate in the very nature of the transaction process, like the irregular spacing between observations ${ }^{1}$, the discrete price changes between observations ${ }^{2}$, or the bounce effect ${ }^{3}$ induced by trading costs in the form of a bid-ask spread. Others are attributed to the process of information diffusion and price discovery like serial dependency found in many variables which are supposed to contain information about the state of the price process, like the intensity of the price process ${ }^{4}$, i.e. the time between price changes, the variation of transaction costs, or the volume traded in a certain time span. In spite of the important role these variables usually play when market microstructure is assessed, they are usually considered as nuisance in the context of risk analysis and the analysis of large scale price changes. ${ }^{5}$ The standard way to proceed would be to define a particular aggregation scheme which supposedly does away with these effects. Andersen and Bollerslev (1998b) show that the choice of an appropriate aggregation scheme is very crucial for these models. By choosing different aggregation levels they obtain strongly different estimates for the volatility per hour. They show that volatility forecasts can be improved significantly by determining an adequate aggretation level and by considering effects on various frequency levels. In this context they illustrate that the volatility process is driven by the simultaneous interaction of different patterns at the intradaily and daily level. These results demonstrate that the choice of the aggregation scheme has a strong impact on the volatility estimation.

\footnotetext{
${ }^{1}$ See the seminal paper of Engle (1996) or Engle and Russell (1997) for a statistical analysis.

${ }^{2}$ See e.g. the early rounding models like Harris (1990), Gottlieb and Kalay (1985) or Ball (1988). For an analysis of the economic implications see Harris (1994). Some more recent models take into account discreteness as well as the irregular spacing of observations, e.g. Hausman, Lo, and MacKinlay (1992), Manrique and Shephard (1997), Russell and Engle (1998), or Rydberg and Shephard (1999).

${ }^{3}$ See the original work of Roll (1984) or also Hausman, Lo, and MacKinlay (1992).

${ }^{4}$ See Easley, Kiefer, and O'Hara (1997) for a structural model of the price process.

${ }^{5}$ This is not meant to imply that the relationship between trade frequency, traded volume and volatility as analyzed e.g. by Clark (1973), Gallant, Rossi, and Tauchen (1992), or Jones, Kaul, and Lipson (1994) is not worthwhile. Yet, for the assessment of risk in this context it is only of limited use.
} 
In order to circumvent these imponderabilities we consider an avenue of research initiated by Cho and Frees (1988) and Russell and Engle (1998). They quantify the instantaneous volatility of the price process by modelling price intensities, i.e. the time between price changes. Their main results concentrate on the serial dependencies found in the process of price intensities and the relationship to hypotheses emanating from market microstructure literature.

In this context the volatility is based on the time the price process spends in the neighborhood of a certain price level. The size of this neigborhood is the tuning parameter used to focus the empirical analysis. The strategy pursued is to analyze the time it takes the price process to generate a certain, given price change. Hence, the volatility is determined by the conditional expected duration between two price events, given the explanatory variables. This kind of volatility estimation differs from the classical strategy where the conditional variances are modeled, either over a given interval as in the standard GARCH literature or over irregular time intervals including more or less appropriate corrections for the intensity of the price process. ${ }^{6}$

Our approach circumvents the problems of correcting for the irregularity of time intervals by using these durations to model volatility patterns directly. By using a semiparametric proportional hazard model we estimate the conditional probability for a price event in the next time interval, given the time the last price event dates back. These conditional probabilities can be interpreted as the risk for price changes in the next time interval. Based on these probabilities we calculate a conditional volatility, which solves the problem of an appropriate aggregation level by defining explicitly the event of interest, too. Hence, the corresponding time intervals and thereby the frequency of observations are determined by the definition of a price event. This feature is the most important advantage of this approach. As will be shown in the course of this paper, this redefinition of

\footnotetext{
${ }^{6}$ Ghysels and Jasiak (1997) develop a class of ARCH models for transaction data which are unequally spaced in time. They combine a temporal aggregation procedure for GARCH models and ACD models for the duration between particular trades. In this bivariate ACDGARCH model they are able to test for Granger causal relationships between the volatility and intra-trade durations. Grammig and Wellner (1999) provide an extension of this approach.
} 
observations is a straightforward solution for most problems induced by market microstructure.

By analyzing price events at the LIFFE Bund Future market we have to take into account that this market is not open $24 \mathrm{~h}$ per day, i.e. spells can also end at the next trading day or during the night. This feature leads by definition to a grouping of the durations caused by the fact that price events occuring overnight are not observable. Hence, we use a categorical model based on Han and Hausman (1990) which allows us to consider grouped durations. ${ }^{7}$ In this framework the $\log$ likelihood is formed by using differences in the survivor function, thus, the model corresponds to an ordered response approach so that the baseline hazard can be calculated by the estimated thresholds. The particular advantage of this approach is that price events occurring overnight can be taken into account. Such price changes can only be observed at the next morning, hence the exact length of the spell cannot be measured. Because we know exactly when the spells started, but do not know when they finished these observations enter the log likelihood by lower and upper bounds. In the following we call these observations 'pseudo censored'.

A test for serial correlation is based on generalized residuals and allows to check for misspecifications. A further advantage of this model is that it permits us to check for unobservable heterogeneity. In the literature on duration a wide range of investigations exists which analyze the impact of misspecified heterogeneity effects on the estimated baseline hazard $\operatorname{rate}^{8}$. In this model unobservable heterogeneity is specified parametrically by a gamma distributed random variable entering the hazard multiplicatively leading to a mixture model based on the BurrII distribution.

A further question which needs to be resolved is the assessment of seasonalities. Intraday seasonalities are a well documented fact ${ }^{9}$ and have to be take into account when estimating intraday volatilities. In the context of futures trading

\footnotetext{
${ }^{7}$ Hautsch (1999) uses this framework to analyze the time between particular trades on the LIFFE Bund Future market, where grouping structures can also be observed.

${ }^{8}$ See e.g. Heckman and Singer (1984).

${ }^{9}$ See e.g. Wood, McInish, and Ord (1985) and McInish and Wood (1992) for an analysis of seasonalities of trading at the NYSE, or Dacorogna, Müller, Nagler, Olsen, and Pictet (1993)
} 
a second source of seasonalities becomes apparent, namely the time to maturity. Both types of seasonalities are analyzed in this paper using a Fourier series expansion suggested by Andersen and Bollerslev (1997).

The outline of our paper is as follows: Section 2 discusses the estimation of volatilities and the inclusion of seasonalities. Section 3 describes the econometric model. Data is described in section 4. Empirical findings are given in section 5. Section 6 presents the conclusions.

\section{Volatility Estimates Based on the Intensity of the Price}

\section{PROCESS}

2.1. A New Measure of Volatility. In order to give a concise picture of the relationship between the new risk estimation concept proposed here and concepts proposed in previous work, it seems worthwhile to look at the standard definition of conditional volatility per time $\operatorname{as}^{10}$

$$
\begin{aligned}
\sigma_{t}^{2}=E\left[\frac{1}{\Delta t}\left(r_{t}-\bar{r}_{t}\right)^{2} \mid X_{t-1}\right] \\
\text { with } r_{t}:=\frac{p(t)-p(t-1)}{p(t-1)}, \\
\bar{r}_{t}=E\left[r_{t} \mid X_{t-1}\right]=0 \\
\text { and } \Delta t_{t}:=t_{N(t)+1}-t_{N(t)} .
\end{aligned}
$$

Here, $t$ denotes the clock time, measured e.g. in seconds since a reference date and $N(t)$ the number of transactions observed up to $t, X$ contains a set of at least weakly exogeneous regressors. In order to apply standard GARCH-type procedures to the estimation problem in eq. (1) $\Delta t$ is usually assumed to be constant. Hence, intra-day aggregates are typically used, e.g. on the basis of 5 minute intervals. See e.g. Andersen and Bollerslev (1998a) and Andersen, Bollerslev, Diebold, and Labys (1999) for further references. Eq. (1) is quite unprecise with respect to the measure which is used within the integral. As it

and Guillaume, Dacorogna, Dave, Müller, Olsen, and Pictet (1997) for the foreign exchange market, or Andersen and Bollerslev (1997) for an in depth analysis of intraday periodicity.

${ }^{10}$ For the ease of exposition no $\log$ returns are used. However, using log returns would not raise any substantial problems. 
is clear from the preceeding argument in the context of standard GARCH-type models the price intensity is considered deterministic and constant over all $t$ :

$$
\sigma_{t,(r)}^{2}=E_{r}\left[r_{t}^{2} \mid X_{t-1}\right] \frac{1}{\Delta t}
$$

In order to account for seasonalities some modifications are needed. This can be done by an inclusion of particular explanatory variables $X_{t}$ as suggested by Andersen and Bollerslev (1997), see also section 3.2, by the introduction of time varying coefficients as in Bollerslev and Ghysels (1996), or by the introduction of concepts of time deformation in the spirit of Dacorogna, Gauvreau, Müller, Olsen, and Pictet (1996). Such a procedure, however raises the question of an optimal aggregation level as a major problem which has to be solved before estimators of this type can be used for risk assessment. Evidently, there are two main sources of errors introduced. First, it is an unresolved issue what the criteria of optimality are for the determination of the aggregation level. See the discussion of Andersen and Bollerslev (1998a) in the context of a continuous time DGP. Second, it is far from clear what kind of bias is introduced by a constant aggregation level particularly for assets having a 'liquidity life cycle' as is the case for a future.

Some models specify indeed a bivariate process, which accounts for the stochastic nature of both, the process of price changes and the process of price intensities. These specifications allow volatility estimates on the basis of the bivariate distribution. With the random variable $d(t):=p(t)-p(t-1)$ we have thus

$$
\sigma_{t,(d, \Delta t)}^{2}=E_{d, \Delta t}\left[\frac{1}{\Delta t} \cdot d(t)^{2} \mid X_{t-1}\right] \frac{1}{p(t-1)^{2}} .
$$

The limitation of these models to the analysis of price changes $d(t)$ is standard and not substantial, considering that these models are usually estimated on the basis of transaction data. Furthermore, it does not limit the applicability given that this factor can be recovered ex post. Having already emphasized that these models are based on transaction data the process of returns, or price changes has a peculiar property which needs to be accounted for. Price changes take on only a few different values depending on the tick size and liquidity of the traded asset. 
For an analysis of the effects of neglected discreteness see Harris (1990), Gottlieb and Kalay (1985), or Ball (1988). Most models concentrating on the bivariate process account explicitly for those market microstructure effects, like Russell and Engle (1998) or Rydberg and Shephard (1999). Ghysels and Jasiak (1997) and Grammig and Wellner (1999) analyze a bivariate process including returns in a GARCH specification which accounts explicitly for the stochastic nature of the price intensity. Nevertheless they refrain from taking market microstructure effects into account. However, the forementioned procedures yield valuable information for the analysis of market microstructure but are of limited use for risk assessment since it is a tedious task to aggregate these models to obtain a valid risk estimator and again, the results of these models mainly reflect the structure of the price process as measured from one transaction to the next, but yield only a limited amount of information for the overall risk of a series.

In the context of the model we propose, we start with the assumption that a decision maker in need of a risk measure is indeed able to express the size of a significant price change. We call this size $\alpha$. Using this $\alpha$ in the given context we recognize that the risk measure boils down to the question of how long it might take to realize this significant price change, i.e. the empirical analysis of first passage times. The bivariate distribution of $r$ and $\Delta t$ is no longer of interest since $r$ is reduced to the ratio of a constant and a conditionally deterministic variable. Note that $\Delta t$ is redefinde as the duration to complete a price change of size $\alpha$. So, we can formulate a conditional volatility $\sigma^{* 2}\left(t \mid X_{t}\right)$ per time $\operatorname{as}^{11}$

$$
\begin{aligned}
\sigma_{t,(\Delta t)}^{* 2} & =E_{\Delta t}\left[\frac{1}{\Delta t} \mid X_{t-1}\right]\left(\frac{\alpha}{p(t-1)}\right)^{2} \\
& =\sigma_{t}^{2} \cdot \frac{1}{p(t-1)^{2}} .
\end{aligned}
$$

This procedure has a second significant benefit that for typical values of $\alpha$ a data dependent aggregation scheme is obtained, which rids the researcher of many market microstructure effects which are a nuisance in the analysis of risk.

$\sigma_{t}^{2}$ stands for the conditional volatility of price changes from which the conditional volatility of returns can easily be recovered (eq. 5). For ease of exposition

\footnotetext{
${ }^{11}$ For a similar specification of a marginal volatility see Engle and Russell (1998).
} 
and without implying a limitation of the suggested procedure, only the volatility of price changes is considered from here on. $X_{t-1}$ includes the covariates' information up to time $t-1, \alpha$ is the size of price changes being analyzed, and $p(t-1)$ the price at time $t-1$.

\section{ThE ECONOMETRIC APPROACH}

Our strategy of volatility estimation is based on the time between certain price events. Thus, it is necessary to model the rate of price changes, i.e. the price intensities. This rate of price changes allows us to measure the speed of price adjustments (see also Engle and Russell (1997)). Furthermore, it provides valuable insights into the information content of price durations. If one investigates durations in financial markets one has to account for the occurrence of event clustering. Short respectively large durations are followed by durations of the same kind which has already been found in different empirical investigations (see e.g. Engle and Russell (1995), Engle and Russell (1997), Engle and Russell (1998), Bollerslev and Domowitz (1993), or Andersen and Bollerslev (1997)). To take into account autocorrelations between durations, Engle and Russell propose the Autoregressive Conditional Duration (ACD) model which specifies the time flow directly via an autoregressive process. This type of models is based on the expected duration between events. By dividing each duration by their conditional expectation capturing the past trading history, 'standardized' durations are obtained which are assumed to be i.i.d. The work of Engle and Russell was the starting point for a wide range of investigations in the class of ACD models. Ghysels and Jasiak (1997) combine the ACD and GARCH models by a joint structure of the volatility of the price process and the durations. Bauwens and Giot (1998b) introduce a logarithmic ACD model which allows for more flexibility. Furthermore they propose an extension of the ACD model by considering the state of prices at hte beginning and the end of each duration (Bauwens and Giot (1998a)). Grammig, Hujer, Kokot, and Maurer (1998) develop a further member of the class of ACD models by proposing an approach which is based on the Burr-distribution. By assuming that the 'standardized' durations follow a 
Burr-distribution the Burr-ACD model nests the Exponential-ACD (EACD) and Weibull-ACD (WACD) model as special cases. Jasiak (1999) proposes a fractional ACD model to account for longer run dependencies potentially contained in the data.

However, these higly versatile ACD models cannot be directly applied to the given problem for two reasons. First, by the definition our spells may extend over one day. The non-trading period overnight introduces a strong structure on our data which needs to be accounted for. Second, some spells are 'pseudo-censored', i.e. we may know exactly when they started, but we do not know exactly when they were finished, we can just be sure that a price change occured overnight. To take into account these grouping structures of durations we employ a model for grouped durations. The specification is based on the approaches of Han and Hausman (1990) and Meyer (1990) and allows the simultaneous estimation of the coefficients of explanatory variables and of a nonparametric baseline survivor function. By examining the intraday and interday transaction price process we define $t_{i}, i=1, \ldots, N$ as the recorded timing of transactions with price events ${ }^{12}$ and $\Delta t_{i}=t_{i}-t_{i-1}$ the duration between these price events, which is assumed to be a random variable following a nonspecified distribution. The model is based on the proportional hazard specification of Cox (1972):

$$
\lambda\left(\Delta t_{i} \mid X_{i}\right)=\lambda_{0}\left(\Delta t_{i}\right) \exp \left(-X_{i}^{\prime} \beta\right)
$$

where $X_{i}$ denotes a $R \times 1$-vector of covariates, $\beta$ a $R \times 1$-vector of coefficients and $\lambda_{0}$ a nonspecified baseline hazard rate corresponding to the hazard rate measured at $X_{i}=0$. Following Kiefer (1988) or Han and Hausman (1990) the proportional hazard model admits a convenient interpretation as a linear model

$$
\tau_{i}=X_{i}^{\prime} \beta+e_{i}
$$

where

$$
\tau_{i} \equiv \ln \left[\Lambda_{0}\left(\Delta t_{i}\right)\right]
$$

\footnotetext{
${ }^{12} \mathrm{~A}$ price event is the time it takes the process to realize a significant price change. See section 4 for details.
} 
and $\Lambda_{0}\left(\Delta t_{i}\right)$ denotes the integrated baseline hazard. In this specification the error term $e_{i}$ follows an extreme value distribution of type $1^{13}$ and does not depend on $\Lambda_{0}\left(\Delta t_{i}\right)$. For the error terms conditional independence is assumed, given the covariates $X_{i}$.

Using (7) as latent model the specification corresponds to an ordered response model. A semiparametric baseline hazard is obtained which can be calculated by the estimated thresholds of the categories. We partition the durations in $K+1$ categories, where we use the durations $\overline{\Delta t_{k}}, k=1, \ldots, K$ as category bounds. The thresholds of the latent model are defined by

$$
\delta_{k} \equiv \ln \left[\Lambda_{0}\left(\overline{\Delta t_{k}}\right)\right]
$$

The conditional probability of failure, i.e. the occurrence of a trade, in category $\mathrm{k}$, conditioned on $X_{i}$ is

$$
\operatorname{Pr}\left[\overline{\Delta t_{k-1}}<\Delta t_{i} \leq \overline{\Delta t_{k}} \mid X_{i}\right]=\int_{\delta_{k-1}-X_{i}^{\prime} \beta}^{\delta_{k}-X_{i}^{\prime} \beta} f(s) d s,
$$

where $f(s)$ denotes the density function of the extreme value distribution.

It has to be taken into account that a price change which occurs within the night cannot be measured exactly because the exact timing of the event is not observable. We only can observe the time from the previous price change until the close which is the minimum of the real price intensity. The price change is observable at the earliest at the opening of the next trading day, hence the time from the last price event at the previous trading day until the next morning is the maximal price intensity. We denote such observations as 'pseudo-censored' observations, for which only a lower bound, $\Delta t_{\min }$ and an upper bound $\Delta t_{\max }$ can be recorded. Using an indicator variable $c_{i}$, denoting the occurrence of pseudocensored observations,

$$
c_{i}=\left\{\begin{array}{lll}
1 & , \quad \Delta t_{i} \in\left[\Delta t_{\min , i} ; \Delta t_{\max , i}\right] \\
0 & , \text { else }
\end{array}\right.
$$

and defining $\delta_{j} \equiv \ln \left[\Lambda_{0}\left(\overline{\Delta t_{j}}\right)\right], j \in\{l, u\}$ as the thresholds so that $\overline{\Delta t_{l}}\left(\overline{\Delta t_{u}}\right)$ is the lower (upper) bound of the category containing $\Delta t_{\min , i}\left(\Delta t_{\max , i}\right)$. The log

\footnotetext{
${ }^{13}$ See Johnson, Kotz, and Balakrishnan (1994).
} 
likelihood function takes the form

(10)

$l(\beta, \delta)=\sum_{i=1}^{N} \sum_{k=1}^{K} \mathbf{1}\left\{\Delta t_{i} \in\left(\overline{\Delta t_{k-1}}, \overline{\Delta t_{k}}\right]\right\} \ln \int_{\delta_{k-1}-X_{i}^{\prime} \beta}^{\delta_{k}-X_{i}^{\prime} \beta} f(s) d s+\sum_{i=1}^{N} c_{i} \ln \int_{\delta_{l}-X_{i}^{\prime} \beta}^{\delta_{u}-X_{i}^{\prime} \beta} f(s) d s$.

In this formula the last term denotes the probability to observe a pseudo-censored price intensity between the observable lower and upper duration bound.

The semiparametric baseline survivor function can be directly obtained through the estimated thresholds. It can be calculated at $k$ discrete points by

$$
S_{0}\left(\overline{\Delta t_{k}}\right)=\exp \left(-\exp \left(\delta t_{k}\right)\right), \quad k=1, \ldots, K
$$

Furthermore, this model allows to account for unobservable heterogeneity effects. Lancaster (1979) and Heckman and Singer (1984) show that ignoring unobserved heterogeneity can lead to biased estimates of the hazard function. Lancaster (1994) shows that the specification of unobservable heterogeneity in the hazard function can capture errors in recorded durations or regressors.

In econometric duration literature two main directions exist concerning the implementation of unobservable heterogeneity. Heckman and Singer (1984), Honore (1990) and Bearse, Canals, and Rilstone (1994) use nonparametric specifications for heterogeneity effects, but also need parametric forms for the baseline hazard rate. On the other hand, Cox (1972), Kiefer (1988), Han and Hausman (1990) and Meyer (1990) include non-parametric baseline hazards but specify unobservable heterogeneity parametrically ${ }^{14}$.

Along the lines of Han and Hausman (1990) we specify heterogeneity effects using a parametric form by a random variable which enters the hazard function multiplicatively. An important advantage of this approach is that the survivor function of the compounded model can be calculated in closed form and that it does not require numerical integration. Including a gamma distributed random variable $\omega$ with $E[\omega]=1$ and $\operatorname{Var}[\omega]=\theta^{-1}$ which acts multiplicatively with the hazard function, a mixed proportional hazard model is obtained. It is assumed

\footnotetext{
${ }^{14} \mathrm{An}(1998)$ proposes consistent estimators for grouped durations which allow both the baseline hazard function and the distribution of the unobserved heterogeneity to be flexible.
} 
that $\omega$ is independent of $X_{i}$. Given the covariates and unobservable heterogeneity effects, the conditional hazard is equal to

$$
\lambda\left(\Delta t_{i} \mid X_{i}, \omega\right)=\lambda_{0}\left(\Delta t_{i}\right) \exp \left(-X_{i}^{\prime} \beta\right) \cdot \omega_{i}, \quad i=1, \ldots, N
$$

This mixture duration model also can interpreted as a linear model ${ }^{15}$

$$
\tau_{i}=X_{i}^{\prime} \beta+\ln (\theta)-e_{i}
$$

where $e_{i}$ follows an $\operatorname{BurrII}(\theta)$ distribution ${ }^{16}$. The baseline survivor function is obtained by

$$
S_{0}\left(\overline{\Delta t_{k}}\right)=\frac{1}{\left[1+\exp \left(\delta_{k}-\ln (\theta)\right)\right]^{\theta}}, \quad k=1, \ldots, K .
$$

It is easily shown that this model converges to an extreme value distribution if $\theta^{-1}=\operatorname{Var}(\omega) \rightarrow 0$. Hence, if no unobservable effects exist, the compounded model corresponds to the extreme value specification in eq. (7).

The use of the described model of grouped durations becomes apparent, if one reformulates $\sigma^{2}\left(t \mid X_{t-1}\right)$ as

$$
\begin{aligned}
\sigma^{2}\left(t \mid X_{t-1}\right)= & \alpha^{2} \sum_{k=1}^{K+1} \operatorname{Prob}\left[\Delta \mathrm{t} \in\left[\overline{\Delta \mathrm{t}_{\mathrm{k}-1}}, \overline{\Delta \mathrm{t}_{\mathrm{k}}}\right) \mid \mathrm{X}_{\mathrm{t}-1}\right] \\
& \cdot \mathrm{E}\left[\frac{1}{\Delta \mathrm{t}} \mid \Delta \mathrm{t} \in\left[\overline{\Delta \mathrm{t}_{\mathrm{k}-1}}, \overline{\Delta \mathrm{t}_{\mathrm{k}}}\right), \mathrm{X}_{\mathrm{t}-1}\right]
\end{aligned}
$$

and notes that in the context of grouped durations the approximation

$$
\mathrm{E}\left[\frac{1}{\Delta \mathrm{t}} \mid \Delta \mathrm{t} \in\left[\overline{\Delta \mathrm{t}_{\mathrm{k}-1}}, \overline{\Delta \mathrm{t}_{\mathrm{k}}}\right), \mathrm{X}_{\mathrm{t}-1}\right] \approx \mathrm{E}\left[\frac{1}{\Delta \mathrm{t}} \mid \Delta \mathrm{t} \in\left[\overline{\Delta \mathrm{t}_{\mathrm{k}-1}}, \overline{\Delta \mathrm{t}_{\mathrm{k}}}\right)\right]
$$

must be valid so that there is an obvious sample estimator for the second factor in the weighted sum of eq. (15). This does not exclude the conditioning information, but merely expresses the fact that all information contained in the explanatory variables enters only the first factor Prob $\left[\Delta t \in\left[\overline{\Delta t_{k-1}}, \overline{\Delta t_{k}}\right) \mid X_{t-1}\right]$ for which an estimator is readily available using the grouped proportional hazard model, cf. (eq. 9).

An important assumption in this ordered response approach is the conditional independence of the error terms. All serial dependence has to be captured by the $X_{i}$ leading to conditionally i.i.d. error terms. To check for this assumption we

\footnotetext{
${ }^{15}$ For details see Hautsch (1999).

${ }^{16}$ See Johnson, Kotz, and Balakrishnan (1994).
} 
use the concept of generalized residuals proposed by Gourieroux, Monfort, and Trognon (1987) to obtain a test for serial correlation based on the extreme value distribution. ${ }^{17}$

To check the goodness of fit of the regression we use the pseudo- $R^{2}$ proposed by McKelvey and Zavoina (1975).

\section{DATA}

4.1. The Generation of Price Events. Our sample contains intraday data of the LIFFE Bund Future trading. Within the observation period the Bund Future was one of the most actively traded future contracts in Europe and was a notional 6\% German government bond of DEM 250.000 face value which matured in 8.5 to 10.5 years at contract expiration. There were four contract maturities per year, March, June, September and December. Prices were denoted in basis points of face value, thus one tick was equivalent to a contract value of DEM 25. This study uses data on 11 contracts and 816 trading days between 5/9/94 and $6 / 5 / 97$ with a total of 2,054,807 transactions which amounts to about 2,500 transactions per day.

The dataset contains time stamped prices, bids, asks and volumes associated with the transaction. The price series consists of $N$ arrival times and $N$ prices $\left\{\left(t_{1}, p_{1}\right), \ldots,\left(t_{N}, t p_{N}\right)\right\}$. From these pairs price durations are obtained by thinning the process and selecting only some of the pairs. Thus, waiting times between price changes are obtained by the time between the points which are kept. Similar to Engle and Russell (1997) the retained series can be defined formally as follows:

1. Retain the first point of the point process (point 1).

2. Retain point $j>1$ if $a b s\left(p_{j}-p_{r}\right)>\alpha$, where $r$ is the index of the most recent retained point.

$\alpha$ is constant and can be chosen arbitrarily. In the empirical analysis we use values of $\alpha \in\{5,10,15,20\}$, corresponding to $27621,7314,3458$, and 2159 observations

\footnotetext{
${ }^{17}$ For details see Hautsch (1999), who provided a test for serial correlation based on the BurrII distribution.
} 
(see table 1 for details). We consider price events occurring within the first 5 minutes of a trading day to be price changes which occurred overnight. Note that we do not use the first 10 minutes of a trading day to avoid the special opening phase. This is reasonable because at LIFFE trading often shows erratic price changes whithin the first trading minutes which even out just after a few transactions. These price changes are due to the price finding process at the opening of each trading day which is based on news releases occuring overnight. We assume that price events occurring after the first 15 minutes of one trading day are driven by recent information, thus, for these observations the exact waiting time can be measured. Figure 1 shows the arrival times of three transactions occuring on different trading days. Thus, based on figure 1, the 'pseudo-censored' duration between $\mathrm{A}$ and $\mathrm{B}$ is at least 15 minutes and at most 15 hours and 30 minutes, whereby the waiting times between $\mathrm{A}$ and $\mathrm{C}$ are exactly 16 hours.

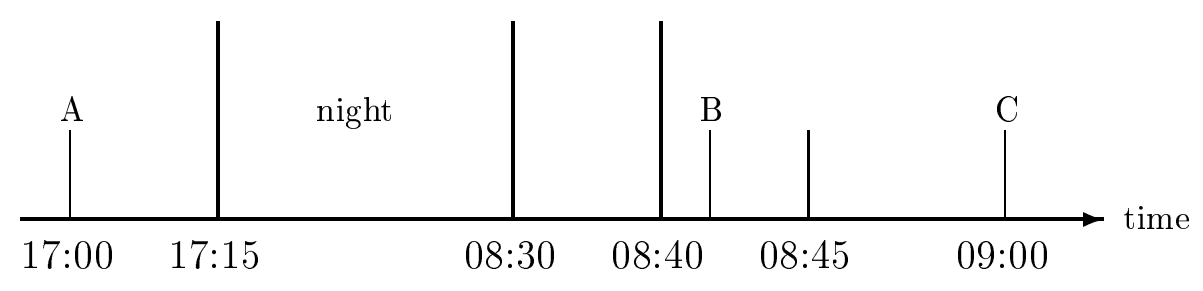

Figure 1: Recorded arrival times of price changes which occur overnight.

By using 5, 10, 15 and 20 tick durations we obtain 680, 516, 372, and 267 'pseudocensored' durations.

The dependent variable is obtained from the categorized price durations. We choose a categorization which ensures satisfactory frequencies of observations in each category. We divide one trading day, corresponding to 8 hours 45 minutes, in 12 categories and the following trading day in two categories. Table 2 and 3 in the appendix show the categorization and the distribution of the categorized durations for the different price changes.

4.2. The Inclusion of Seasonalities. Intradaily seasonalities of the volatility are a well known problem in empirical research of market microstructure, see 
e.g. Wood, McInish, and Ord (1985) or Müller, Dacorogna, Dave, Olsen, Pictet, and Weizsäcker (1997). Engle and Russell (1997) present strong empirical evidence for seasonalities in price durations. One solution is to generate seasonally adjusted series by partialling out the time-of-day effects. Engle and Russell (1997) regress the duration on a function of the time and obtain an estimator of the typical shape. By dividing the durations by their estimated typical shape they obtain the seasonally adjusted series.

Here, a Fourier series approximation proposed by Andersen and Bollerslev (1998b) based on the work of Gallant (1981) is employed. This framework allows for a direct but parsimonious assessment of the volatility patterns based on different frequencies, like intraday seasonalities in contrast to time to maturity seasonalities. Additional regressors of the form $t^{*}, \cos \left(t^{*} \cdot 2 \pi p\right)$, and $\sin \left(t^{*} \cdot 2 \pi p\right)$ are included in the regression, where $t^{*} \in[0,1]$ and $p$ is identical with the order of the term. The total effect of seasonalities with coefficients $\delta_{1}, \delta_{c, p}$, and $\delta_{s, p}$ and order of approximation $P$ is given by

$$
s\left(\delta, t^{*}, P\right)=\delta_{1} \cdot t^{*}+\sum_{p=1}^{P}\left(\delta_{c, p} \cos \left(t^{*} \cdot 2 \pi p\right)+\delta_{s, p} \sin \left(t^{*} \cdot 2 \pi p\right)\right) .
$$

For the analysis of intraday seasonalities

$$
t_{1}^{*}=\frac{\text { seconds since } 8: 45}{\text { seconds between 8:45 and 17:15 }}
$$

is used as a reference and for the analysis of time to maturity effects

$$
t_{2}^{*}=\frac{\text { days to maturity }}{160} .
$$

By using terms up to order 5 it is posssible to estimate volatility patterns on different frequency levels. The days to maturity regressors are based on a sample length of 160 days. To account for observations with values of more than 160 days to maturity we include a dummy variable.

\section{EMPiRical Findings}

5.1. Estimation Results. Tables 4-7 in the appendix give the results for the duration estimation using 5,10,15 and 20 tick price changes. For each tick size we estimate three specifications, one model without any explanatory variables (A), 
one regression only with intraday seasonalities (B) and one specification with all seasonalities $(\mathrm{C})$.

For all four different price durations it is shown that the goodness of fit is significantly improved by the inclusion of time to maturity seasonalities. Looking at the pseudo $R^{2}$ values one can see that the explanatory power of the days to maturity seasonalities seems to increase for larger price changes while the explanatory power of included intraday patterns seem to decrease slightly. Hence, the larger the analyzed price changes the higher the impact of the lower frequent days to maturity seasonality effects while the strength of intradaily patterns weakens.

Most of the intraday and days to maturity seasonalities' coefficients are significant on a $5 \%$ level, whereas the significance decreases slightly for higher price changes caused by smaller sample sizes.

Based on all analyzed price durations we find very similar results for the estimated coefficients, i.e. seasonal patterns seem not to depend on the chosen price event $^{18}$.

Table 8 gives the calculated values of the baseline survivor function $S_{0}$ and the conditional probabilities $\lambda_{0}^{*}$ for the occurrence of an event in a certain category based on the estimated thresholds for 15 tick and 20 tick durations. ${ }^{19}$ Note that this discrete baseline hazard rate $\lambda_{0}\left(\overline{\Delta t_{k}}\right)$ is evaluated as

$$
\lambda_{0}\left(\overline{\Delta t_{k}}\right)=\frac{S_{0}\left(\overline{\Delta t_{k}}\right)-S_{0}\left(\overline{\Delta t_{k+1}}\right)}{S_{0}\left(\overline{\Delta t_{k}}\right)} \cdot \frac{1}{\overline{\Delta t_{k+1}}-\overline{\Delta t_{k}}}, \quad k=1, \ldots, K,
$$

so that the resulting values are only comparable if the width of the categories are identicaly. Thus, it is reasonable to use the conditional 'failure probability', which can be interpreted directly as a risk measure,

$$
\lambda_{0}^{*}\left(\overline{\Delta t_{k}}\right)=P\left(\overline{\Delta t_{k}} \leq \Delta t_{i}<\overline{\Delta t_{k+1}} \mid \Delta t_{i} \geq \overline{\Delta t_{k}}\right)=\frac{S_{0}\left(\overline{\Delta t_{k}}\right)-S_{0}\left(\overline{\Delta t_{k+1}}\right)}{S_{0}\left(\overline{\Delta t_{k}}\right)} .
$$

It is shown that the baseline survivor function based on 15 tick price durations decreases faster than for 20 ticks due to the fact that 20 tick price durations are

\footnotetext{
${ }^{18}$ Note that with the proportinal hazard specification of eq. 6 , we have $\partial \lambda\left(\Delta t_{i} \mid X_{i}\right) / \partial X_{i}=-\beta$, i.e. a positive sign indicates higher durations and thus lower volatilities.

${ }^{19}$ These baseline functions are calculated based on mean values for all explanatory variables, i.e. they correspond to the baseline survivor functions and conditional probabilities for an 'average' observation.
} 
larger. The probabilities for surviving certain time horizons are also higher. This feature is also reflected in the corresponding baseline failure probabilities leading to lower values for the 20 tick price durations. By analyzing these values it has to taken into account that the particular interval lengths differ. The wider the categories, the higher must be the conditional failure probabilities. Nevertheles these values can be used to quantify the risk for price changes within certain time intervals and can be used as risk measures.

5.2. Seasonalities over the Future's Maturity. A reasonable interpretation of the days to maturity seasonalities requires looking at all regressors within the Fourier series approximation ${ }^{20}$. Based on this flexible form we calculate the price change volatility $\sigma^{2}\left(t \mid X_{t}\right)$ based on all significant $(5 \%$ level) coefficients. Figure 2 shows the pattern of $\sigma^{2}\left(t \mid X_{t}\right)$ depending on the time to maturity for 20 tick price changes, while the intraday seasonal coefficients are fixed on a value corresponding to $14: 00$.

We find the highest peak at a position of about 90 days until maturity. In the Bund Future market there are expiration days every three months. These high trading activities are caused by the roll-over from the previous contract to the front month contract and might thus indicate a special type of liquidity effect. The highest volatility values are found within a time horizon between 80 and 90 days to maturity followed by a decreasing pattern which might be explained by the beginning of the next roll-over period from this contract to the following front month contract or the fact that the stochastic properties of the future converge to the underlying asset with decreasing time to maturity.

The results found for 20 tick price changes can be confirmed by analyzing the volatility patterns based on the other price change durations. For all samples we find a significant peak at approximately 90 days with a somewhat decreasing shape at the end of the Future's maturity.

5.3. Intraday Seasonalities. Figure 3 depicts the seasonal patterns of the intraday price change volatility for a fixed time to maturity of 16 days based on

\footnotetext{
${ }^{20}$ The interpretation of single coefficients is not reasonable because the seasonality patterns are based on the sum of all included terms.
} 
20 tick price durations. The figures indicate a volatility shape with an increasing pattern after the opening, a low volatility at 12:00 and further peaks at 13:00 and 14:30. Thus, after the opening the volatility increases and remains almost constant within the morning. At 12:00 there is a lunch break which reduces the volatility. After the lunch break a significant increasing volatility pattern is observable, followed by a little 'dip' at 13:30. Before the beginning of U.S. trading at 14:30 market participants seem to reduce their trading activites and 'wait' for news from American traders which enter the market at this point in time. Furthermore, the beginning of U.S. trading at 14:30 has a strong impact on the price change volatility, indicated by the highest volatility level on the trading day. After that the volatility strongly decreases until 15:30.

By investigating other price durations we are confirming these results. All volatility patterns indicate intradaily seasonalities with a significant 'dip' at the lunch break and a peak at 14:30. Hence, these results seem to be robust and do not depend on the chosen price event and, thus, the aggregation level.

5.4. Serial Correlation and Heterogeneity. To check for misspecification due to serial dependence in the error terms we use a test on serial correlation based on Gourieroux, Monfort, and Trognon (1987). Tables 9-11 show the test statistics based on the regressions for 5, 10, 15 and 20 tick durations for all three models A,B and C. Comparing the test statistics of the different specifications it is apparent that the intraday and days to maturity seasonalities capture serial correlation structures. Thus, serial dependencies are also caused by seasonalities on different frequency levels. This confirms the results of Engle and Russell (1997) who also show that serial correlation in price durations can be reduced by seasonal adjustments.

Furthermore, we find that the test statistics are lower for larger price changes considered, i.e. the larger the investigated price durations the weaker the clustering structures. Based on 5 tick price changes we find extremely high test statistics for all three specifications indicating the existence of serial correlations. While for 10 tick price changes (specification C) slight serial dependencies in the errors 
are found, these effects diminish for 15 ticks and 20 ticks. Furthermore, these results support the assumption of conditional independence of the error terms given the covariates.

To check for unobservable heterogeneity effects, we also examine a gamma compounded hazard model (see table 12). Heterogeneity increases the larger the price changes. For 20 ticks we find for the heterogeneity variance a value of 0.9048 , i.e. this specification almost corresponds to an ordered logit approach ${ }^{21}$. The increasing heterogeneity effects may be caused by the stronger impact of unobservable news effects. The relative importance of macro news which is not captured by explanatory variables increases the larger the analyzed price changes and, also, the smaller the sample size. This feature coincides with the results of Hautsch (1999) who obtained only very small heterogeneity effects by analyzing transaction data. Preliminary studies on the basis of individual contracts support the hypothesis that unobservable heterogeneity is caused by news effects. This is clearly an issue for further research. A thorough analysis is clearly beyond the scope of this paper.

To check the impact of heterogeneity we also estimate volatilities based on the regressions of table 12 . The figures 4 and 5 show the resulting volatility patterns. Due the additional heterogeneity the intradaily and time to maturity seasonality patterns are more pronounced, while the fundamental pattern is nearly unaffected. This indicates that the estimated volatilities are very robust against misspecifications.

\section{Conclusions}

This paper investigates the use of price intensities to estimate volatilities based on high-frequency transaction data. In this context we analyze the durations between significant price events. These price events are defined as the time it takes to complete a price change of a certain tick size which is exogeneously given. Such durations allow us to measure the speed of price adjustments so that the conditional probability for a price change in a certain time interval

\footnotetext{
${ }^{21}$ Note that the BurrII(1) distribution corresponds to the logistic distribution.
} 
can be interpreted as a risk measure. Using these price durations in order to estimate volatilities solves the problem of an appropriate aggregation level by defining explicitly the event of interest, hence, the frequency of observations is determined automatically by the definition of the price event.

The fact that the LIFFE Bund Future market is not open $24 \mathrm{~h}$ a day leads to a grouping of the durations since price events occuring overnight are not observable. For this reason we use a categorical model based on Han and Hausman (1990). Duration categories are considered, where the log likelihood is evaluated by using differences in the survivor function. This model allows us to take into account price changes occurring overnight which can not be measured exactly. We only know when the spells started, but do not know exactly when they were finished. Thus, only a lower and an upper bound of the duration is obtained.

We use a dataset containing LIFFE Bund Future transactions of 11 contracts. To take intraday seasonalities and time to maturity seasonalities into account we include a Fourier series approximation proposed by Andersen and Bollerslev (1998b) which allows for an assessment of volatility patterns based on different frequencies.

It is shown that the goodness of fit is significantly improved by the inclusion of time to maturity seasonalities, where the explanatory power of these variables seem to increase the larger the analyzed price durations are. Estimated thresholds allow us to calculate the conditional probabilities for the occurrence of a price event within a certain time horizon.

We find a significant peak in the volatility around 90 days to maturity, caused by the roll-over from the previous contract to the front month contract. The shape of intraday price change volatilities provides empirical evidence for the well-known daily trading patterns with an increasing pattern after the opening, a decreasing volatility at the lunch break and a significant peak at the opening of U.S. trading at 14:30.

To check for misspecification due to serial correlation in the error terms we use a test based on generalized residuals. Two main results are obtained: First, intraday and days to maturity seasonalities play an important role by capturing 
serial dependencies. Second, we find strong serial correlations for lower price durations which diminish the larger the exogeneous size of price changes is. For 15 and 20 ticks no empirical evidence for the existence of serial correlation is found.

The inclusion of unobservable heterogeneity allows us to check for misspecifications like missing variables or errors in the dependent and independent variables. It can be seen that heterogeneity increases the larger the analyzed price changes are. This may be caused by stronger impacts of unobservable news effects in the context of smaller sample sizes. The analysis of these potential news effects indicates a promising avenue of further research. 


\section{REFERENCES}

An, M. Y. (1998): "Consistent Estimation of Regression Coefficients in Mixed Proportional Hazard Models," Discussion Paper June, Paper Presented at the Conference on Panel Data and Structural Labor Market Models, Sandbjerg, DK.

Andersen, T. G., And T. Bollerslev (1997): "Intraday Periodicity and Volatility Persistence in Financial Markets," Journal of Empirical Finance, 4, 115-158.

(1998a): "Answering the Skeptics: Yes, Standard Volatility Models Do Provide Accurate Forecasts," International Economic Review, 39(6023), 885-905.

- (1998b): "Deutsche Mark-Dollar Volatility: Intraday Activity Patterns, Macroeconomic Announcements, and Longer Run Dependencies," Journal of Finance, 53, 219-265.

Andersen, T. G., T. Bollerslev, F. X. Diebold, And P. Labys (1999): "The Distribution of the Exchange Rate Volatility," Discussion Paper 6961, NBER Working Paper Series.

Ball, C. (1988): "Estimation Bias Induced by Discrete Security Prices," Journal of Finance, $43,841-865$.

Bauwens, L., AND P. Giot (1998a): "Asymmetric ACD Models: Introducing Price Information in ACD Models with a Two State Transition Model," Discussion Paper 9844, CORE. (1998b): "The Logarithmic ACD Model: An Applicatioin to Market Microstructure and NASDAQ," Discussion Paper 9789, CORE.

Bearse, P., J. Canals, And P. Rilstone (1994): "Semiparametric Maximum Likelihood Estimation of Duration Models with Unobserved Heterogeneity," Discussion paper, Department of Economics, York University.

Bollerslev, T., AND I. Domowitz (1993): "Trading Patterns and Prices in the Interbank Foreign Exchange Market," Journal of Finance, 48, 1421-1443.

Bollerslev, T., AND E. Ghysels (1996): "Periodic Autoregressive Conditional Heteroscedasticity," Journal of Business and Economic Statistics, 14, 139-151.

Cho, D. C., AND E. W. Frees (1988): "Estimating the Volatility of Discrete Stock Prices," Journal of Finance, 43, 451-466.

Clark, P. K. (1973): "A Subordinated Stochastic Process Model with Finite Variance for Speculative Prices," Econometrica, 41, 135-155.

Cox, D. R. (1972): "Regression Models and Life-Tables," Journal of the Royal Statistical Society, Series B, 34, 187-200.

Dacorogna, M. M., C. L. Gauvreau, U. A. Müller, R. B. Olsen, and O. V. Pictet (1996): "Changing Time Scale for Short-term Forecasting in Financial Markets," Journal of Forecasting, 15, 203-227.

Dacorogna, M. M., U. A. Müller, R. J. Nagler, R. B. Olden, and O. V. Pictet (1993): "A Geographical Model for the Daily and Weekly Seasonal Volatility in the Foreign Exchange Market," Journal of International Money and Finance, 12, 413-438.

Easley, D., N. M. Kiefer, AND M. O'Hara (1997): "The Information Content of the Trading Process," Journal of Empirical Finance, 4, 159-186.

Engle, R. F. (1996): "The Econometrics of Ultra-High Frequency Data," Discussion paper, Department of Economics, UCSD.

Engle, R. F., AND J. R. Russell (1995): "Forecasting Transaction Rates: The Autoregressive Conditional Duration," Discussion paper, UCSD.

Engle, R. F., AND J. R. Russell (1997): "Forecasting the Frequency of Changes in Quoted Foreign Exchange Prices with the Autoregressive Conditional Duration Model," Journal of Empirical Finance, 4, 187-212.

- (1998): "Autoregressive Conditional Duration: A New Model for Irregularly Spaced Transaction Data," Econometrica, 66, 1127-1162.

Gallant, A. R., P. E. Rossi, And G. Tauchen (1992): "Stock Prizes and Volume," Review of Financial Studies, 5, 199-242. 
Gallant, R. A. (1981): "On the Bias in Flexible Functional Forms and an Essential Unbiased Form: The Fourier Flexible Form," Journal of Econometrics, 15, 211-245.

Ghysels, E., AND J. JAsiak (1997): "GARCH for Irregularly Spaced Financial Data: The ACD-GARCH Model," Discussion Paper 97s-06, CIRANO, Montreal.

Gottlieb, G., AND A. Kalay (1985): "Implications of the Discreteness of Observed Stock Prices," Journal of Finance, 40, 135-154.

Gourieroux, C., A. Monfort, And A. Trognon (1987): "Generalized Residuals," Journal of Econometrics, 34, 5-32.

Grammig, J., R. Hujer, S. Kokot, And K.-O. Maurer (1998): "Modeling the Deutsche Telekom IPO Using a New ACD Specification. An Application of the Burr-ACD Model Using High-Frequency Ibis Data," Discussion paper, Humboldt-Universität, Berlin.

Grammig, J., AND M. Wellner (1999): "Modeling the Interdependence of Volatility and Inter-Transaction Duration Processes," Discussion Paper, Humboldt-University, Berlin, 21.

Guillaume, D. M., M. M. Dacorogna, R. R. Dave, U. A. Müller, R. B. Olsen, And O. V. Pictet (1997): "From the Bird's Eye to the Microscope: A Survey of New Stylized Facts of the Intra-Daily Foreign Exchange Markets," Finance and Stochastics, 1, 95-129.

Han, A., AND J. A. Hausman (1990): "Flexible Parametric Estimation of Duration and Competing Risk Models," Journal of Applied Econometrics, 5, 1-28.

HARRIS, L. (1990): "Estimation of Stock Variances and Serial Covariances from Discrete Observations," Journal of Financial and Quantitative Analysis, 25, 291-306.

Harris, L. E. (1994): "Minimum Price Variations, Discrete Bid-Ask Spreads, and Quotation Sizes," Review of Financial Studies, 7, 149-178.

Hausman, J. A., A. W. Lo, And A. C. MacKinlay (1992): "An Ordered Probit Analysis of Transaction Stock Prices," Journal of Financial Economics, 31, 319-379.

Hautsch, N. (1999): "Analyzing the Time Between Trades with a Gamma Compounded Hazard Model. An Application to LIFFE Bund Future Transctions," Discussion Paper 99/03, CoFE, Konstanz.

Heckman, J., AND B. Singer (1984): "Econometric Duration Analyses," Journal of Econometrics, 24, 63-132.

Honore, B. E. (1990): "Simple Estimation of a Duration Model with Unobserved Heterogeneity," Econometrica, 58, 453-473.

JASIAK, J. (1999): "Persistence in Intratrade Durations," Discussion paper, York University, Montreal.

Johnson, N. L., S. Kotz, And N. Balakrishnan (1994): Continuous Univariate Distributions, vol. 1. Wiley, New York.

Jones, C. M., G. Kaul, And M. L. Lipson (1994): "Transactions, Volume, and Volatility," Review of Financial Studies, 7, 631-651.

Kiefer, N. M. (1988): "Economic Duration Data and Hazard Functions," Journal of Economic Literature, 26, 646-679.

Lancaster, T. (1979): "Econometric Methods for the Duration of Unemployment," Econometrica, 47, 939-956.

Lancaster, T. (1994): The Econometric Analysis of Transition Data. Cambridge University Press, New York.

Manrique, A., And N. Shephard (1997): "Likelihood Analysis of a Discrete Bid/Ask Price Model for a Common Stock Quoted on the NYSE," Discussion paper, Nuffield College, Oxford University.

McInish, T., AND R. Wood (1992): "An Analysis of Intraday Patterns in Bid/Ask Spreads for NYSE Stocks," Journal of Finance, 47, 753-764.

McKelvey, R., And W. Zavoina (1975): "A Statistical Model for the Analysis of Ordinal Level Dependent Variables," Journal of Mathematical Sociology, 4, 103-120.

MEYER, B. (1990): "Unemployment Insurance and Unemployment Spells," Econometrica, 58, $757-782$. 
Müller, U. A., M. M. Dacorogna, R. R. Dave, R. B. Olden, O. V. Pictet, and J. E. V. Weizsäcker (1997): "Volatilities of Different Time Resolutions - Analyzing the Dynamics of Market Components," Journal of Empirical Finance, 4, 213-239.

RolL, R. (1984): "A Simple Implicit Measure of the Effective Bid-Ask Spread in an Efficient Market," Journal of Finance, 39, 1127-1139.

Russell, J. R., AND R. F. Engle (1998): "Econometric Analysis of Discrete-Valued, Irregularly-Spaced Financial Transactions Data Using a New Autoregressive Conditional Multinomial Model," Discussion paper, presented at Second International Conference on High Frequency Data in Finance, Zurich, Switzerland.

Rydberg, T. H., AND N. Shephard (1999): "Dynamics of Trade-by-Trade Price Movements: Decomposition and Models," Discussion paper, Nuffield College, Oxford, presented at Symposium on Microstructure and High Frequency Data, Paris, France.

Wood, R. A., T. H. McInish, AND J. K. ORD (1985): "An Investigation of Transactions Data for NYSE Stocks," Journal of Finance, 40, 723-741. 
Appendix A. Empirical Results

\section{A.1. Descriptive Statistics.}

TABLE 1. Number of observations per contract and overall observations. Number of pseudo-censored observations per contract and overall. Based on BUND futures trading at LIFFE, London.

\begin{tabular}{lrrrrrrrr}
\hline \multirow{2}{*}{ Contract } & \multicolumn{3}{c}{5 Ticks } & \multicolumn{2}{c}{10 Ticks } & \multicolumn{2}{c}{15 Ticks } & \multicolumn{2}{c}{ Ticks } \\
& Obs. & $\begin{array}{r}\text { Cens. } \\
\text { obs. }\end{array}$ & Obs. & $\begin{array}{r}\text { Cens. } \\
\text { obs. }\end{array}$ & Obs. & $\begin{array}{r}\text { Cens. } \\
\text { obs. }\end{array}$ & $\begin{array}{r}\text { Obs. } \\
\text { obs. }\end{array}$ \\
\hline 1 & 3849 & 65 & 1067 & 54 & 488 & 38 & 334 & 30 \\
2 & 2185 & 55 & 557 & 40 & 284 & 27 & 166 & 16 \\
3 & 2573 & 61 & 655 & 51 & 313 & 38 & 184 & 26 \\
4 & 2616 & 63 & 704 & 52 & 311 & 33 & 203 & 31 \\
5 & 1929 & 65 & 482 & 39 & 215 & 24 & 136 & 15 \\
6 & 3033 & 65 & 777 & 55 & 376 & 41 & 224 & 28 \\
7 & 2738 & 66 & 752 & 59 & 365 & 38 & 230 & 28 \\
8 & 1648 & 57 & 458 & 39 & 214 & 30 & 124 & 16 \\
9 & 1894 & 60 & 479 & 39 & 225 & 28 & 136 & 21 \\
10 & 2642 & 61 & 727 & 43 & 353 & 39 & 206 & 23 \\
11 & 2514 & 62 & 656 & 49 & 314 & 36 & 216 & 33 \\
\hline Total & 27621 & 680 & 7314 & 516 & 3458 & 372 & 2159 & 267 \\
\hline
\end{tabular}

TABle 2. Distribution of categorized price durations based on 5 tick and 10 tick price changes. Based on BUND futures trading at LIFFE, London. Means and Standard Deviations in Seconds. Distribution of the lower bounds of pseudo-censored durations.

\begin{tabular}{lrrrr|rrrr}
\hline & \multicolumn{4}{c}{5 Ticks } & \multicolumn{5}{c}{10 Ticks } \\
Categories & Obs & Mean & Std.Dv. & Cens & Obs & Mean & Std.Dv. & Cens \\
\hline$[0,2 \mathrm{~min}]$ & 4595 & 1.02 & 0.57 & 103 & 297 & 0.98 & 0.56 & 24 \\
$(2 \mathrm{~min}, 4 \mathrm{~min}]$ & 3967 & 2.94 & 0.57 & 92 & 251 & 3.01 & 0.59 & 18 \\
$(4 \mathrm{~min}, 8 \mathrm{~min}]$ & 5118 & 5.84 & 1.14 & 113 & 482 & 6.08 & 1.14 & 43 \\
$(8 \mathrm{~min}, 12 \mathrm{~min}]$ & 3188 & 9.87 & 1.13 & 102 & 441 & 9.99 & 1.16 & 42 \\
$(12 \mathrm{~min}, 20 \mathrm{~min}]$ & 3610 & 15.53 & 2.31 & 106 & 848 & 15.07 & 2.27 & 53 \\
$(20 \mathrm{~min}, 30 \mathrm{~min}]$ & 2226 & 24.40 & 2.89 & 71 & 748 & 24.71 & 2.88 & 65 \\
$(30 \mathrm{~min}, 1 \mathrm{~h}]$ & 2350 & 41.63 & 8.44 & 66 & 1306 & 43.10 & 8.50 & 133 \\
$(1 \mathrm{~h}, 2 \mathrm{~h}]$ & 1113 & 81.88 & 16.24 & 11 & 1096 & 84.31 & 17.09 & 69 \\
$(2 \mathrm{~h}, 3 \mathrm{~h}]$ & 324 & 144.75 & 17.52 & 3 & 464 & 144.74 & 16.86 & 31 \\
$(3 \mathrm{~h}, 4 \mathrm{~h}]$ & 155 & 206.72 & 16.70 & 1 & 267 & 206.82 & 17.66 & 5 \\
$(4 \mathrm{~h}, 6 \mathrm{~h}]$ & 130 & 290.26 & 37.08 & 7 & 297 & 290.83 & 35.33 & 11 \\
$(6 \mathrm{~h}, 8 \mathrm{~h} 45 \mathrm{~min}]$ & 84 & 438.60 & 51.58 & 5 & 193 & 430.51 & 49.17 & 22 \\
$(8 \mathrm{~h} 45 \mathrm{~min}, 17 \mathrm{~h} 15 \mathrm{~min}]$ & 78 & 681.71 & 128.27 & 0 & 105 & 679.68 & 121.66 & 0 \\
$(17 \mathrm{~h} 15 \mathrm{~min}, \infty)$ & 3 & 1065.13 & 23.88 & 0 & 3 & 1065.13 & 23.88 & 0 \\
\hline total & 26941 & 21.79 & 55.82 & 680 & 6798 & 81.69 & 122.83 & 516 \\
\hline
\end{tabular}


TABLE 3. Distribution of categorized price durations based on 15 tick and 20 tick price changes. Based on BUND futures trading at LIFFE, London. Means and Standard Deviations in Seconds. Distribution of the lower bounds of pseudo-censored durations.

\begin{tabular}{lrrrr|rrrr}
\hline & \multicolumn{4}{c}{15 Ticks } & \multicolumn{5}{c}{20 Ticks } \\
Categories & Obs & Mean & Std.Dv. & Cens & Obs & Mean & Std.Dv. & Cens \\
\hline$[0,2 \mathrm{~min}]$ & 44 & 0.97 & 0.50 & 8 & 18 & 0.76 & 0.39 & 5 \\
$(2 \mathrm{~min}, 4 \mathrm{~min}]$ & 41 & 2.98 & 0.54 & 6 & 11 & 2.63 & 0.64 & 2 \\
$(4 \mathrm{~min}, 8 \mathrm{~min}]$ & 82 & 5.92 & 1.16 & 11 & 32 & 6.00 & 1.14 & 4 \\
$(8 \mathrm{~min}, 12 \mathrm{~min}]$ & 89 & 10.15 & 1.19 & 13 & 24 & 10.42 & 1.20 & 9 \\
$(12 \mathrm{~min}, 20 \mathrm{~min}]$ & 173 & 16.15 & 2.33 & 27 & 69 & 16.06 & 2.39 & 15 \\
$(20 \mathrm{~min}, 30 \mathrm{~min}]$ & 210 & 25.02 & 2.92 & 42 & 67 & 25.05 & 3.11 & 25 \\
$(30 \mathrm{~min}, 1 \mathrm{~h}]$ & 527 & 44.47 & 8.63 & 70 & 228 & 44.68 & 8.25 & 40 \\
$(1 \mathrm{~h}, 2 \mathrm{~h}]$ & 590 & 85.79 & 16.88 & 75 & 324 & 88.83 & 16.99 & 57 \\
$(2 \mathrm{~h}, 3 \mathrm{~h}]$ & 307 & 147.83 & 16.67 & 28 & 211 & 150.35 & 15.90 & 28 \\
$(3 \mathrm{~h}, 4 \mathrm{~h}]$ & 233 & 207.87 & 17.83 & 9 & 144 & 206.69 & 17.15 & 13 \\
$(4 \mathrm{~h}, 6 \mathrm{~h}]$ & 339 & 295.46 & 33.89 & 26 & 258 & 298.49 & 35.91 & 18 \\
$(6 \mathrm{~h}, 8 \mathrm{~h} \mathrm{45min}]$ & 285 & 437.60 & 47.77 & 57 & 266 & 439.21 & 47.73 & 51 \\
$(8 \mathrm{~h} 45 \mathrm{~min}, 17 \mathrm{~h} 15 \mathrm{~min}]$ & 163 & 669.43 & 120.67 & 0 & 237 & 685.58 & 131.70 & 0 \\
$(17 \mathrm{~h} 15 \mathrm{~min}, \infty)$ & 3 & 1065.13 & 23.88 & 0 & 3 & 1065.13 & 1040.95 & 0 \\
\hline total & 3086 & 166.83 & 180.85 & 372 & 1892 & 244.97 & 224.01 & 267 \\
\hline
\end{tabular}




\section{A.2. Five Tick Price Changes.}

TABLE 4. Estimation of proportional hazard models for grouped durations and McKelvey-Zavoina's pseudo $R^{2}$ statistic. Based on BUND futures trading at LIFFE, London using 5 tick price changes. 27,621 observations. P-values based on asymptotic tstatistics.

\begin{tabular}{|c|c|c|c|c|c|c|}
\hline \multirow[b]{2}{*}{ Variable } & \multicolumn{2}{|c|}{ A } & \multicolumn{2}{|c|}{ B } & \multicolumn{2}{|c|}{ C } \\
\hline & Coeff. & p-value & Coeff. & $\mathrm{p}$-value & Coeff. & $\mathrm{p}$-value \\
\hline \multicolumn{7}{|c|}{ Thresholds } \\
\hline$\mu_{1}$ & -1.7000 & 0.0000 & -1.8179 & 0.0000 & -2.5054 & 0.0000 \\
\hline$\mu_{2}$ & -0.9846 & 0.0000 & -1.0945 & 0.0000 & -1.7725 & 0.0000 \\
\hline$\mu_{3}$ & -0.3675 & 0.0000 & -0.4671 & 0.0000 & -1.1297 & 0.0000 \\
\hline$\mu_{4}$ & -0.0395 & 0.0000 & -0.1310 & 0.0000 & -0.7798 & 0.0000 \\
\hline$\mu_{5}$ & 0.3339 & 0.0000 & 0.2560 & 0.0000 & -0.3666 & 0.0000 \\
\hline$\mu_{6}$ & 0.5915 & 0.0000 & 0.5273 & 0.0000 & -0.0644 & 0.0004 \\
\hline$\mu_{7}$ & 0.9438 & 0.0000 & 0.9056 & 0.0000 & 0.3950 & 0.0000 \\
\hline$\mu_{8}$ & 1.1919 & 0.0000 & 1.1723 & 0.0000 & 0.7755 & 0.0000 \\
\hline$\mu_{9}$ & 1.2942 & 0.0000 & 1.2786 & 0.0000 & 0.9513 & 0.0000 \\
\hline$\mu_{10}$ & 1.3402 & 0.0000 & 1.3247 & 0.0000 & 1.0339 & 0.0000 \\
\hline$\mu_{11}$ & 1.3922 & 0.0000 & 1.3753 & 0.0000 & 1.1268 & 0.0000 \\
\hline$\mu_{12}$ & 1.6445 & 0.0000 & 1.6211 & 0.0000 & 1.6178 & 0.0000 \\
\hline \multicolumn{7}{|c|}{ Intraday Seasonalities } \\
\hline$\delta_{1}$ & & & 0.4109 & 0.0000 & 0.4825 & 0.0000 \\
\hline$\delta_{s, 1}$ & & & 0.3712 & 0.0000 & 0.4374 & 0.0000 \\
\hline$\delta_{s, 2}$ & & & 0.0115 & 0.1832 & 0.0290 & 0.0212 \\
\hline$\delta_{s, 3}$ & & & -0.0232 & 0.0134 & -0.0216 & 0.0305 \\
\hline$\delta_{s, 4}$ & & & 0.1087 & 0.0000 & 0.1113 & 0.0000 \\
\hline$\delta_{s, 5}$ & & & -0.0548 & 0.0000 & -0.0619 & 0.0000 \\
\hline$\delta_{c, 1}$ & & & -0.1836 & 0.0000 & -0.2047 & 0.0000 \\
\hline$\delta_{c, 2}$ & & & 0.1089 & 0.0000 & 0.1307 & 0.0000 \\
\hline$\delta_{c, 3}$ & & & -0.0258 & 0.0023 & -0.0117 & 0.1162 \\
\hline$\delta_{c, 4}$ & & & 0.0732 & 0.0000 & 0.0949 & 0.0000 \\
\hline$\delta_{c, 5}$ & & & 0.0936 & 0.0000 & 0.1040 & 0.0000 \\
\hline \multicolumn{7}{|c|}{ DTM Seasonalities } \\
\hline$\delta_{1}^{*}$ & & & & & 1.4047 & 0.0000 \\
\hline Dummy $_{>160}$ & & & & & 1.8807 & 0.0000 \\
\hline$\delta_{s, 1}^{*}$ & & & & & -0.4958 & 0.0000 \\
\hline$\delta_{s, 2}^{*}$ & & & & & -0.2454 & 0.0000 \\
\hline$\delta_{s, 3}^{*}$ & & & & & -0.0522 & 0.0143 \\
\hline$\delta_{s, 4}^{*}$ & & & & & -0.2023 & 0.0000 \\
\hline$\delta_{s, 5}^{*}$ & & & & & 0.0319 & 0.0043 \\
\hline$\delta_{c, 1}^{*}$ & & & & & 0.6401 & 0.0000 \\
\hline$\delta_{c, 2}^{*}$ & & & & & -0.1989 & 0.0000 \\
\hline$\delta_{c, 3}^{*}$ & & & & & 0.0453 & 0.0196 \\
\hline$\delta_{c, 4}^{*}$ & & & & & -0.1042 & 0.0000 \\
\hline$\delta_{c, 5}^{*}$ & & & & & -0.1136 & 0.0000 \\
\hline \multicolumn{7}{|c|}{ McKelvey-Zavoina's ps. $R^{2}$} \\
\hline & 0.0000 & & 0.0819 & & 0.1350 & \\
\hline
\end{tabular}




\section{A.3. Ten Tick Price Changes.}

TABLE 5. Estimation of proportional hazard models for grouped durations and McKelvey-Zavoina's pseudo $R^{2}$ statistic. Based on BUND futures trading at LIFFE, London, using 10 tick price changes. 7,314 observations. P-values based on asymptotic tstatistics.

\begin{tabular}{|c|c|c|c|c|c|c|}
\hline \multirow[b]{2}{*}{ Variable } & \multicolumn{2}{|c|}{$\mathrm{A}$} & \multicolumn{2}{|c|}{$\mathrm{B}$} & \multicolumn{2}{|c|}{$\mathrm{C}$} \\
\hline & Coeff. & p-value & Coeff. & $\mathrm{p}$-value & Coeff. & $\mathrm{p}$-value \\
\hline \multicolumn{7}{|c|}{ Thresholds } \\
\hline$\mu_{1}$ & -3.1826 & 0.0000 & -3.2662 & 0.0000 & -3.7269 & 0.0000 \\
\hline$\mu_{2}$ & -2.5492 & 0.0000 & -2.6293 & 0.0000 & -3.0867 & 0.0000 \\
\hline$\mu_{3}$ & -1.8768 & 0.0000 & -1.9540 & 0.0000 & -2.4059 & 0.0000 \\
\hline$\mu_{4}$ & -1.4807 & 0.0000 & -1.5561 & 0.0000 & -2.0033 & 0.0000 \\
\hline$\mu_{5}$ & -0.9418 & 0.0000 & -1.0142 & 0.0000 & -1.4517 & 0.0000 \\
\hline$\mu_{6}$ & -0.5827 & 0.0000 & -0.6511 & 0.0000 & -1.0790 & 0.0000 \\
\hline$\mu_{7}$ & -0.0530 & 0.0004 & -0.1080 & 0.0000 & -0.5118 & 0.0000 \\
\hline$\mu_{8}$ & 0.3524 & 0.0000 & 0.3237 & 0.0000 & -0.0377 & 0.0724 \\
\hline$\mu_{9}$ & 0.5395 & 0.0000 & 0.5297 & 0.0000 & 0.2026 & 0.0000 \\
\hline$\mu_{10}$ & 0.6518 & 0.0000 & 0.6524 & 0.0000 & 0.3507 & 0.0000 \\
\hline$\mu_{11}$ & 0.7959 & 0.0000 & 0.8074 & 0.0000 & 0.5469 & 0.0000 \\
\hline$\mu_{12}$ & 0.8603 & 0.0000 & 0.8773 & 0.0000 & 0.6417 & 0.0000 \\
\hline$\mu_{13}$ & 1.2800 & 0.0000 & 1.3242 & 0.0000 & 1.3031 & 0.0000 \\
\hline \multicolumn{7}{|c|}{ Intraday Seasonalities } \\
\hline$\delta_{1}$ & & & 0.8786 & 0.0000 & 0.9176 & 0.0000 \\
\hline$\delta_{s, 1}$ & & & 0.5334 & 0.0000 & 0.5697 & 0.0000 \\
\hline$\delta_{s, 2}$ & & & -0.0226 & 0.1598 & -0.0251 & 0.1385 \\
\hline$\delta_{s, 3}$ & & & 0.0052 & 0.3974 & -0.0144 & 0.2334 \\
\hline$\delta_{s, 4}$ & & & 0.0601 & 0.0008 & 0.0361 & 0.0268 \\
\hline$\delta_{s, 5}$ & & & -0.0481 & 0.0074 & -0.0884 & 0.0000 \\
\hline$\delta_{c, 1}$ & & & 0.0397 & 0.0179 & 0.1170 & 0.0000 \\
\hline$\delta_{c, 2}$ & & & 0.1244 & 0.0000 & 0.1554 & 0.0000 \\
\hline$\delta_{c, 3}$ & & & -0.0065 & 0.3592 & 0.0211 & 0.1156 \\
\hline$\delta_{c, 4}$ & & & 0.0986 & 0.0000 & 0.1150 & 0.0000 \\
\hline$\delta_{c, 5}$ & & & 0.1044 & 0.0000 & 0.1053 & 0.0000 \\
\hline \multicolumn{7}{|c|}{ DTM Seasonalities } \\
\hline$\delta_{1}^{*}$ & & & & & 1.3360 & 0.0000 \\
\hline Dummy $_{>} 160$ & & & & & 1.7803 & 0.0000 \\
\hline$\delta_{s, 1}^{*}$ & & & & & -0.2598 & 0.0028 \\
\hline$\delta_{s, 2}^{*}$ & & & & & -0.1967 & 0.0001 \\
\hline$\delta_{s, 3}^{*}$ & & & & & -0.0144 & 0.3498 \\
\hline$\delta_{s, 4}^{*}$ & & & & & -0.1353 & 0.0000 \\
\hline$\delta_{s, 5}^{*}$ & & & & & 0.0160 & 0.2396 \\
\hline$\delta_{c, 1}^{*}$ & & & & & 0.5525 & 0.0000 \\
\hline$\delta_{c, 2}^{*}$ & & & & & -0.1287 & 0.0000 \\
\hline$\delta_{c, 3}^{*}$ & & & & & 0.0173 & 0.2860 \\
\hline$\delta_{c, 4}^{*}$ & & & & & -0.0617 & 0.0151 \\
\hline$\delta_{c, 5}^{*}$ & & & & & -0.0612 & 0.0043 \\
\hline \multicolumn{7}{|c|}{ McKelvey-Zavoina's ps. $R^{2}$} \\
\hline & 0.0000 & & 0.0541 & & 0.1713 & \\
\hline
\end{tabular}




\section{A.4. Fifteen Tick Price Changes.}

TABLE 6. Estimation of proportional hazard models for grouped durations and McKelvey-Zavoina's pseudo $R^{2}$ statistic. Based on BUND futures trading at LIFFE, London, using 15 tick price changes. 3,458 observations. P-values based on asymptotic tstatistics.

\begin{tabular}{|c|c|c|c|c|c|c|}
\hline \multirow[b]{2}{*}{ Variable } & \multicolumn{2}{|c|}{$\mathrm{A}$} & \multicolumn{2}{|c|}{$\mathrm{B}$} & \multicolumn{2}{|c|}{$\mathrm{C}$} \\
\hline & Coeff. & p-value & Coeff. & $\mathrm{p}$-value & Coeff. & $\mathrm{p}$-value \\
\hline \multicolumn{7}{|c|}{ Thresholds } \\
\hline$\mu_{1}$ & -4.3773 & 0.0000 & -4.4356 & 0.0000 & -4.7968 & 0.0000 \\
\hline$\mu_{2}$ & -3.7008 & 0.0000 & -3.7575 & 0.0000 & -4.1169 & 0.0000 \\
\hline$\mu_{3}$ & -3.0051 & 0.0000 & -3.0599 & 0.0000 & -3.4166 & 0.0000 \\
\hline$\mu_{4}$ & -2.5598 & 0.0000 & -2.6135 & 0.0000 & -2.9678 & 0.0000 \\
\hline$\mu_{5}$ & -2.0086 & 0.0000 & -2.0617 & 0.0000 & -2.4119 & 0.0000 \\
\hline$\mu_{6}$ & -1.5689 & 0.0000 & -1.6222 & 0.0000 & -1.9681 & 0.0000 \\
\hline$\mu_{7}$ & -0.8716 & 0.0000 & -0.9228 & 0.0000 & -1.2568 & 0.0000 \\
\hline$\mu_{8}$ & -0.3808 & 0.0000 & -0.4207 & 0.0000 & -0.7345 & 0.0000 \\
\hline$\mu_{9}$ & -0.1671 & 0.0000 & -0.1950 & 0.0000 & -0.4932 & 0.0000 \\
\hline$\mu_{10}$ & -0.0159 & 0.2481 & -0.0344 & 0.0911 & -0.3184 & 0.0000 \\
\hline$\mu_{11}$ & 0.2196 & 0.0000 & 0.2161 & 0.0000 & -0.0367 & 0.1429 \\
\hline$\mu_{12}$ & 0.3553 & 0.0000 & 0.3649 & 0.0000 & 0.1418 & 0.0000 \\
\hline$\mu_{13}$ & 0.9534 & 0.0000 & 1.0208 & 0.0000 & 0.9854 & 0.0000 \\
\hline \multicolumn{7}{|c|}{ Intraday Seasonalities } \\
\hline$\delta_{1}$ & & & 1.0523 & 0.0000 & 1.0776 & 0.0000 \\
\hline$\delta_{s, 1}$ & & & 0.4599 & 0.0000 & 0.4310 & 0.0000 \\
\hline$\delta_{s, 2}$ & & & -0.0546 & 0.0593 & -0.0703 & 0.0201 \\
\hline$\delta_{s, 3}$ & & & -0.0459 & 0.0810 & -0.1010 & 0.0008 \\
\hline$\delta_{s, 4}$ & & & 0.0930 & 0.0019 & 0.0885 & 0.0032 \\
\hline$\delta_{s, 5}$ & & & -0.0350 & 0.1351 & -0.0541 & 0.0442 \\
\hline$\delta_{c, 1}$ & & & 0.1332 & 0.0000 & 0.2482 & 0.0000 \\
\hline$\delta_{c, 2}$ & & & 0.1334 & 0.0000 & 0.1597 & 0.0000 \\
\hline$\delta_{c, 3}$ & & & -0.0368 & 0.1084 & -0.0141 & 0.3163 \\
\hline$\delta_{c, 4}$ & & & 0.0489 & 0.0515 & 0.0794 & 0.0029 \\
\hline$\delta_{c, 5}$ & & & 0.0734 & 0.0053 & 0.0569 & 0.0229 \\
\hline \multicolumn{7}{|c|}{ DTM Seasonalities } \\
\hline$\delta_{1}^{*}$ & & & & & 1.4295 & 0.0001 \\
\hline Dummy $_{>160}$ & & & & & 1.4777 & 0.0000 \\
\hline$\delta_{s, 1}^{*}$ & & & & & -0.1383 & 0.1261 \\
\hline$\delta_{s, 2}^{*}$ & & & & & -0.1403 & 0.0180 \\
\hline$\delta_{s, 3}^{*}$ & & & & & 0.0322 & 0.2628 \\
\hline$\delta_{s, 4}^{*}$ & & & & & -0.0984 & 0.0080 \\
\hline$\delta_{s, 5}^{*}$ & & & & & -0.0103 & 0.3785 \\
\hline$\delta_{c, 1}^{*}$ & & & & & 0.5515 & 0.0000 \\
\hline$\delta_{c, 2}^{*}$ & & & & & -0.0931 & 0.0118 \\
\hline$\delta_{c, 3}^{*}$ & & & & & 0.0350 & 0.1876 \\
\hline$\delta_{c, 4}^{*}$ & & & & & -0.0433 & 0.1278 \\
\hline$\delta_{c, 5}^{*}$ & & & & & -0.0631 & 0.0276 \\
\hline \multicolumn{7}{|c|}{ McKelvey-Zavoina's ps. $R^{2}$} \\
\hline & 0.0000 & & 0.0598 & & 0.1874 & \\
\hline
\end{tabular}




\section{A.5. Twenty Tick Price Changes.}

TABLE 7. Estimation of proportional hazard models for grouped durations and McKelvey-Zavoina's pseudo $R^{2}$ statistic. Based on BUND futures trading at LIFFE, London, using 20 tick price changes. 2,159 observations. P-values based on asymptotic tstatistics.

\begin{tabular}{|c|c|c|c|c|c|c|}
\hline \multirow[b]{2}{*}{ Variable } & \multicolumn{2}{|c|}{$\mathrm{A}$} & \multicolumn{2}{|c|}{$\mathrm{B}$} & \multicolumn{2}{|c|}{$\mathrm{C}$} \\
\hline & Coeff. & p-value & Coeff. & $\mathrm{p}$-value & Coeff. & $\mathrm{p}$-value \\
\hline \multicolumn{7}{|c|}{ Thresholds } \\
\hline$\mu_{1}$ & -4.8359 & 0.0000 & -4.9075 & 0.0000 & -5.2011 & 0.0000 \\
\hline$\mu_{2}$ & -4.3339 & 0.0000 & -4.4045 & 0.0000 & -4.6970 & 0.0000 \\
\hline$\mu_{3}$ & -3.5626 & 0.0000 & -3.6317 & 0.0000 & -3.9219 & 0.0000 \\
\hline$\mu_{4}$ & -3.2186 & 0.0000 & -3.2872 & 0.0000 & -3.5759 & 0.0000 \\
\hline$\mu_{5}$ & -2.5963 & 0.0000 & -2.6649 & 0.0000 & -2.9505 & 0.0000 \\
\hline$\mu_{6}$ & -2.2190 & 0.0000 & -2.2890 & 0.0000 & -2.5724 & 0.0000 \\
\hline$\mu_{7}$ & -1.4524 & 0.0000 & -1.5247 & 0.0000 & -1.8004 & 0.0000 \\
\hline$\mu_{8}$ & -0.8864 & 0.0000 & -0.9552 & 0.0000 & -1.2173 & 0.0000 \\
\hline$\mu_{9}$ & -0.6409 & 0.0000 & -0.7017 & 0.0000 & -0.9526 & 0.0000 \\
\hline$\mu_{10}$ & -0.4990 & 0.0000 & -0.5538 & 0.0000 & -0.7969 & 0.0000 \\
\hline$\mu_{11}$ & -0.2324 & 0.0000 & -0.2749 & 0.0000 & -0.4983 & 0.0000 \\
\hline$\mu_{12}$ & -0.0198 & 0.2560 & -0.0431 & 0.1134 & -0.2413 & 0.0000 \\
\hline$\mu_{13}$ & 0.6625 & 0.0000 & 0.7003 & 0.0000 & 0.6219 & 0.0000 \\
\hline \multicolumn{7}{|c|}{ Intraday Seasonalities } \\
\hline$\delta_{1}$ & & & 0.9980 & 0.0000 & 0.8671 & 0.0000 \\
\hline$\delta_{s, 1}$ & & & 0.3871 & 0.0000 & 0.2931 & 0.0000 \\
\hline$\delta_{s, 2}$ & & & -0.1498 & 0.0011 & -0.1779 & 0.0002 \\
\hline$\delta_{s, 3}$ & & & 0.0054 & 0.4533 & -0.0570 & 0.1125 \\
\hline$\delta_{s, 4}$ & & & 0.0848 & 0.0297 & 0.0763 & 0.0472 \\
\hline$\delta_{s, 5}$ & & & -0.0722 & 0.0489 & -0.0911 & 0.0209 \\
\hline$\delta_{c, 1}$ & & & 0.0976 & 0.0132 & 0.1796 & 0.0000 \\
\hline$\delta_{c, 2}$ & & & 0.0878 & 0.0169 & 0.1373 & 0.0006 \\
\hline$\delta_{c, 3}$ & & & -0.0549 & 0.0954 & -0.0238 & 0.2902 \\
\hline$\delta_{c, 4}$ & & & 0.0258 & 0.2708 & 0.0054 & 0.4503 \\
\hline$\delta_{c, 5}$ & & & 0.0694 & 0.0372 & 0.0791 & 0.0226 \\
\hline \multicolumn{7}{|c|}{ DTM Seasonalities } \\
\hline$\delta_{1}^{*}$ & & & & & 1.0861 & 0.0056 \\
\hline Dummy $_{>} 160$ & & & & & 1.2981 & 0.0000 \\
\hline$\delta_{s, 1}^{*}$ & & & & & -0.2239 & 0.0558 \\
\hline$\delta_{s, 2}^{*}$ & & & & & -0.1867 & 0.0087 \\
\hline$\delta_{s, 3}^{*}$ & & & & & 0.0832 & 0.0919 \\
\hline$\delta_{s, 4}^{*}$ & & & & & -0.0640 & 0.1062 \\
\hline$\delta_{s, 5}^{*}$ & & & & & -0.0200 & 0.3180 \\
\hline$\delta_{c, 1}^{*}$ & & & & & 0.5046 & 0.0000 \\
\hline$\delta_{c, 2}^{*}$ & & & & & -0.0888 & 0.0388 \\
\hline$\delta_{c, 3}^{*}$ & & & & & 0.0113 & 0.4049 \\
\hline$\delta_{c, 4}^{*}$ & & & & & -0.0083 & 0.4298 \\
\hline$\delta_{c, 5}^{*}$ & & & & & -0.0183 & 0.3346 \\
\hline \multicolumn{7}{|c|}{ McKelvey-Zavoina's ps. $R^{2}$} \\
\hline & 0.0000 & & 0.0618 & & 0.1824 & \\
\hline
\end{tabular}




\section{A.6. Baseline Survivor Function.}

TABLE 8. Estimated baseline survivor function $\left(S_{0}\right)$ and conditional baseline failure probabilities $\left(\lambda_{0}^{*}\right)$ based on estimated thresholds from table 6 and 7 columns $(\mathrm{C})$.

\begin{tabular}{|c|c|c|c|c|}
\hline & \multicolumn{2}{|c|}{15 ticks } & \multicolumn{2}{|c|}{20 ticks } \\
\hline Categories & $\overline{S_{0}}$ & $\lambda_{0}^{*}$ & $\overline{S_{0}}$ & $\lambda_{0}^{*}$ \\
\hline$[0,2 \mathrm{~min}]$ & 0.9426 & 0.0573 & 0.9710 & 0.0289 \\
\hline$(2 \mathrm{~min}, 4 \mathrm{~min}]$ & 0.8900 & 0.0558 & 0.9525 & 0.0190 \\
\hline$(4 \mathrm{~min}, 8 \mathrm{~min}]$ & 0.7908 & 0.1114 & 0.8997 & 0.0553 \\
\hline$(8 \mathrm{~min}, 12 \mathrm{~min}]$ & 0.6924 & 0.1244 & 0.8613 & 0.0427 \\
\hline$(12 \mathrm{~min}, 20 \mathrm{~min}]$ & 0.5269 & 0.2390 & 0.7565 & 0.1216 \\
\hline$(20 \mathrm{~min}, 30 \mathrm{~min}]$ & 0.3683 & 0.3008 & 0.6655 & 0.1203 \\
\hline (30min, $1 \mathrm{~h}]$ & 0.1308 & 0.6448 & 0.4143 & 0.3774 \\
\hline$(1 \mathrm{~h}, 2 \mathrm{~h}]$ & 0.0324 & 0.7521 & 0.2062 & 0.5021 \\
\hline$(2 \mathrm{~h}, 3 \mathrm{~h}]$ & 0.0127 & 0.6077 & 0.1278 & 0.3801 \\
\hline$(3 \mathrm{~h}, 4 \mathrm{~h}]$ & 0.0055 & 0.5655 & 0.0904 & 0.2928 \\
\hline$(4 \mathrm{~h}, 6 \mathrm{~h}]$ & 0.0010 & 0.8157 & 0.0391 & 0.5666 \\
\hline$(6 \mathrm{~h}, 8 \mathrm{~h} 45 \mathrm{~min}]$ & 0.0003 & 0.7398 & 0.0151 & 0.6130 \\
\hline (8h $45 \mathrm{~min}, 17 \mathrm{~h} 15 \mathrm{~min}]$ & 0.0000 & 0.9999 & 0.0001 & 0.9967 \\
\hline
\end{tabular}

\section{A.7. Tests on Serial Correlation.}

TABle 9. Test of regressions in tables 4, 5, 6, and 7 columns (A) on misspecification due to serial correlation based on generalized residuals, lags $1-10 . H_{0}$ : No serial correlation at lag $i\left(\xi_{i}\right)$. Critical values at $1 \%, 5 \%$, and $10 \%$ level: 6.635, 3.841, and 2.706 .

\begin{tabular}{lcrrr}
\hline & 5 Ticks & 10 Ticks & 15 Ticks & 20 Ticks \\
\hline$\xi_{1}$ & 5014.7978 & 287.8774 & 19.4954 & 2.0592 \\
$\xi_{2}$ & 3863.1592 & 238.5695 & 6.9822 & 0.0949 \\
$\xi_{3}$ & 3315.2351 & 194.5703 & 17.9820 & 2.3695 \\
$\xi_{4}$ & 2834.9796 & 100.4819 & 4.9803 & 0.3619 \\
$\xi_{5}$ & 2373.9764 & 22.1823 & 1.5386 & 0.1235 \\
$\xi_{6}$ & 2364.4271 & 123.0322 & 2.5090 & 2.1324 \\
$\xi_{7}$ & 2139.8003 & 123.4222 & 2.2497 & 0.5721 \\
$\xi_{8}$ & 1891.9382 & 102.3425 & 4.3821 & 0.0306 \\
$\xi_{9}$ & 1721.7059 & 105.9418 & 6.8202 & 0.0498 \\
$\xi_{10}$ & 1641.0436 & 61.5008 & 0.4659 & 0.1612 \\
\hline
\end{tabular}


TABlE 10. Test of regressions in tables 4, 5, 6, and 7 columns (B) on misspecification due to serial correlation based on generalized residuals, lags 1-10. $H_{0}$ : No serial correlation at lag $i\left(\xi_{i}\right)$. Critical values at $1 \%, 5 \%$, and $10 \%$ level: 6.635, 3.841, and 2.706 .

\begin{tabular}{lcrrr}
\hline & 5 Ticks & 10 Ticks & 15 Ticks & 20 Ticks \\
\hline$\xi_{1}$ & 5449.8539 & 62.8042 & 11.4721 & 1.2000 \\
$\xi_{2}$ & 4490.7992 & 79.5436 & 6.0358 & 0.7104 \\
$\xi_{3}$ & 3873.9594 & 90.6242 & 15.3474 & 3.6648 \\
$\xi_{4}$ & 3302.6187 & 37.6827 & 5.4397 & 0.0647 \\
$\xi_{5}$ & 2991.8758 & 0.6009 & 1.3601 & 1.5913 \\
$\xi_{6}$ & 2835.0514 & 29.6433 & 3.5423 & 3.7501 \\
$\xi_{7}$ & 2498.5187 & 60.3371 & 2.8869 & 2.0328 \\
$\xi_{8}$ & 2192.8304 & 48.4808 & 4.5630 & 0.6801 \\
$\xi_{9}$ & 2050.2449 & 33.6504 & 7.2355 & 0.1519 \\
$\xi_{10}$ & 1888.8035 & 20.2231 & 1.2183 & 0.0249 \\
\hline
\end{tabular}

TABLE 11. Test of regressions in tables 4, 5, 6, and 7 columns (C) on misspecification due to serial correlation based on generalized residuals, lags $1-10 . H_{0}$ : No serial correlation at lag $i\left(\xi_{i}\right)$. Critical values at $1 \%, 5 \%$, and $10 \%$ level: $6.635,3.841$, and 2.706 .

\begin{tabular}{lrrrr}
\hline & \multicolumn{1}{c}{ 5 Ticks } & 10 Ticks & 15 Ticks & 20 Ticks \\
\hline$\xi_{1}$ & 1938.0992 & 3.9901 & 1.5625 & 0.0296 \\
$\xi_{2}$ & 1346.2270 & 9.4565 & 0.1713 & 0.0318 \\
$\xi_{3}$ & 1228.9598 & 23.1374 & 5.8314 & 0.3820 \\
$\xi_{4}$ & 953.7975 & 6.2726 & 0.0307 & 0.6073 \\
$\xi_{5}$ & 794.9280 & 0.2373 & 0.4589 & 0.0097 \\
$\xi_{6}$ & 873.1944 & 3.5244 & 0.0760 & 0.3918 \\
$\xi_{7}$ & 757.0924 & 6.9110 & 0.0590 & 0.2562 \\
$\xi_{8}$ & 632.6383 & 2.8212 & 0.0981 & 0.0144 \\
$\xi_{9}$ & 542.9378 & 3.3464 & 0.4975 & 0.1996 \\
$\xi_{10}$ & 506.3134 & 0.3862 & 0.0931 & 0.6334 \\
\hline
\end{tabular}




\section{A.8. Regressions with Unobserved Heterogeneity.}

TABLE 12. Estimation of gamma compounded proportional hazard models for grouped durations and McKelvey-Zavoina's pseudo $R^{2}$ statistic. Based on BUND futures trading at LIFFE, London, June 1995 contract using 5, 10, 15, and 20 tick price changes. P-values based on asymptotic t-statistics.

\begin{tabular}{|c|c|c|c|c|c|c|c|c|}
\hline \multirow[b]{2}{*}{ Variable } & \multicolumn{2}{|c|}{5 Ticks } & \multicolumn{2}{|c|}{10 Ticks } & \multicolumn{2}{|c|}{15 Ticks } & \multirow{2}{*}{\multicolumn{2}{|c|}{20 Ticks }} \\
\hline & Coeff. & p-value & Coeff. & p-value & Coeff. & p-value & & \\
\hline \multicolumn{9}{|c|}{ Thresholds } \\
\hline$\mu_{1}$ & -2.9795 & 0.0000 & -4.0779 & 0.0000 & -5.1526 & 0.0000 & -5.5652 & 0.0000 \\
\hline$\mu_{2}$ & -2.1747 & 0.0000 & -3.4191 & 0.0000 & -4.4605 & 0.0000 & -5.0511 & 0.0000 \\
\hline$\mu_{3}$ & -1.4160 & 0.0000 & -2.7055 & 0.0000 & -3.7404 & 0.0000 & -4.2549 & 0.0000 \\
\hline$\mu_{4}$ & -0.9685 & 0.0000 & -2.2722 & 0.0000 & -3.2730 & 0.0000 & -3.8958 & 0.0000 \\
\hline$\mu_{5}$ & -0.3934 & 0.0000 & -1.6564 & 0.0000 & -2.6833 & 0.0000 & -3.2394 & 0.0005 \\
\hline$\mu_{6}$ & 0.0674 & 0.0228 & -1.2186 & 0.0000 & -2.1999 & 0.0000 & -2.8373 & 0.0000 \\
\hline$\mu_{7}$ & 0.8410 & 0.0000 & -0.4949 & 0.0000 & -1.3769 & 0.0000 & -1.9792 & 0.0000 \\
\hline$\mu_{8}$ & 1.5480 & 0.0000 & 0.1737 & 0.0006 & -0.70254 & 0.0000 & -1.2617 & 0.0000 \\
\hline$\mu_{9}$ & 1.8930 & 0.0000 & 0.5324 & 0.0000 & -0.3679 & 0.0000 & -0.9067 & 0.0000 \\
\hline$\mu_{10}$ & 2.0576 & 0.0000 & 0.7623 & 0.0000 & -0.1167 & 0.0000 & -0.6891 & 0.0000 \\
\hline$\mu_{11}$ & 2.2082 & 0.0000 & 1.0801 & 0.0000 & 0.3088 & 0.0000 & -0.2506 & 0.0043 \\
\hline$\mu_{12}$ & 2.2429 & 0.0000 & 1.2373 & 0.0000 & 0.5903 & 0.0000 & 0.1509 & 0.0978 \\
\hline$\mu_{13}$ & 3.1898 & 0.0000 & 2.3991 & 0.0000 & 2.0837 & 0.0000 & 1.7338 & 0.0000 \\
\hline \multicolumn{9}{|c|}{ Intraday Seasonalities } \\
\hline$\overline{\delta_{1}}$ & -0.0857 & 0.1862 & 1.3442 & 0.0000 & 1.8099 & 0.0000 & 1.5286 & 0.0000 \\
\hline$\delta_{s, 1}$ & 0.3800 & 0.0000 & 0.8843 & 0.0000 & 0.8585 & 0.0000 & 0.6547 & 0.0000 \\
\hline$\delta_{s, 2}$ & -0.0901 & 0.0000 & 0.0213 & 0.2696 & -0.0102 & 0.4311 & -0.2266 & 0.0011 \\
\hline$\delta_{s, 3}$ & -0.1131 & 0.0003 & 0.0279 & 0.1636 & -0.1369 & 0.0014 & -0.1535 & 0.0127 \\
\hline$\delta_{s, 4}$ & 0.1475 & 0.0000 & 0.0915 & 0.0006 & 0.1504 & 0.0006 & 0.1010 & 0.0572 \\
\hline$\delta_{s, 5}$ & -0.1316 & 0.0000 & -0.1202 & 0.0000 & -0.1296 & 0.0029 & -0.1823 & 0.0052 \\
\hline$\delta_{c, 1}$ & -0.3363 & 0.0000 & 0.0570 & 0.0302 & 0.3250 & 0.0000 & 0.3679 & 0.0000 \\
\hline$\delta_{c, 2}$ & 0.1583 & 0.0000 & 0.2348 & 0.0000 & 0.3424 & 0.0000 & 0.3197 & 0.0000 \\
\hline$\delta_{c, 3}$ & -0.0821 & 0.0000 & 0.0469 & 0.0384 & 0.0337 & 0.2214 & -0.0405 & 0.2544 \\
\hline$\delta_{c, 4}$ & 0.0918 & 0.0000 & 0.0 .2102 & 0.0000 & 0.1615 & 0.0009 & 0.0665 & 0.1605 \\
\hline$\delta_{c, 5}$ & 0.1275 & 0.0007 & 0.2113 & 0.0000 & 0.1316 & 0.0024 & 0.1384 & 0.0088 \\
\hline \multicolumn{9}{|c|}{ DTM Seasonalities } \\
\hline$\delta_{1}^{*}$ & 2.5942 & 0.0000 & 2.1565 & 0.0006 & 2.6113 & 0.0003 & 2.3147 & 0.0014 \\
\hline Dummy $_{>160}$ & 3.0831 & 0.0000 & 2.6322 & 0.0000 & 2.2854 & 0.0000 & 2.0507 & 0.0000 \\
\hline$\delta_{s, 1}^{*}$ & -0.7091 & 0.0086 & -0.3040 & 0.0190 & -0.0397 & 0.4186 & -0.1370 & 0.2831 \\
\hline$\delta_{s, 2}^{*}$ & -0.3601 & 0.0024 & -0.2586 & 0.0003 & -0.1541 & 0.0652 & -0.2257 & 0.0369 \\
\hline$\delta_{s, 3}^{*}$ & -0.0406 & 0.1278 & -0.0052 & 0.4618 & 0.0887 & 0.1221 & 0.1915 & 0.0285 \\
\hline$\delta_{s, 4}^{*}$ & -0.2904 & 0.0000 & -0.1814 & 0.0000 & -0.1203 & 0.0212 & -0.0605 & 0.2277 \\
\hline$\delta_{s, 5}^{*}$ & 0.0303 & 0.0419 & 0.0230 & 0.2257 & 0.0134 & 0.3879 & 0.0297 & 0.3287 \\
\hline$\delta_{c, 1}^{*}$ & 1.0430 & 0.0000 & 0.7989 & 0.0000 & 0.8194 & 0.0000 & 0.7926 & 0.0000 \\
\hline$\delta_{c, 2}^{*}$ & -0.3264 & 0.0000 & -0.1998 & 0.0000 & -0.1411 & 0.0140 & -0.1671 & 0.0185 \\
\hline$\delta_{c, 3}^{*}$ & 0.0756 & 0.0193 & 0.0390 & 0.1989 & 0.0485 & 0.2132 & 0.0058 & 0.4682 \\
\hline$\delta_{c, 4}^{*}$ & -0.1313 & 0.0000 & -0.0911 & 0.0166 & -0.0656 & 0.1292 & -0.0356 & 0.3128 \\
\hline$\delta_{c, 5}^{*}$ & -0.1780 & 0.0000 & -0.0906 & 0.0037 & -0.0597 & 0.1092 & -0.0154 & 0.4062 \\
\hline \multicolumn{9}{|c|}{ Heterogeneity Variance } \\
\hline$V[\omega]$ & 0.5210 & 0.0000 & 0.5204 & 0.0000 & 0.6886 & 0.0000 & 0.9048 & 0.0000 \\
\hline \multicolumn{9}{|c|}{$\overline{\text { McKelvey-Zavoina's ps. } R^{2}}$} \\
\hline & 0.2405 & & 0.2414 & & 0.2617 & & 0.2524 & \\
\hline
\end{tabular}




\section{APPENDiX B. FiguRES}

Figure 2: 20 tick Price Change Volatility vs. DTM, 14:00 BUND Future trading, LIFFE

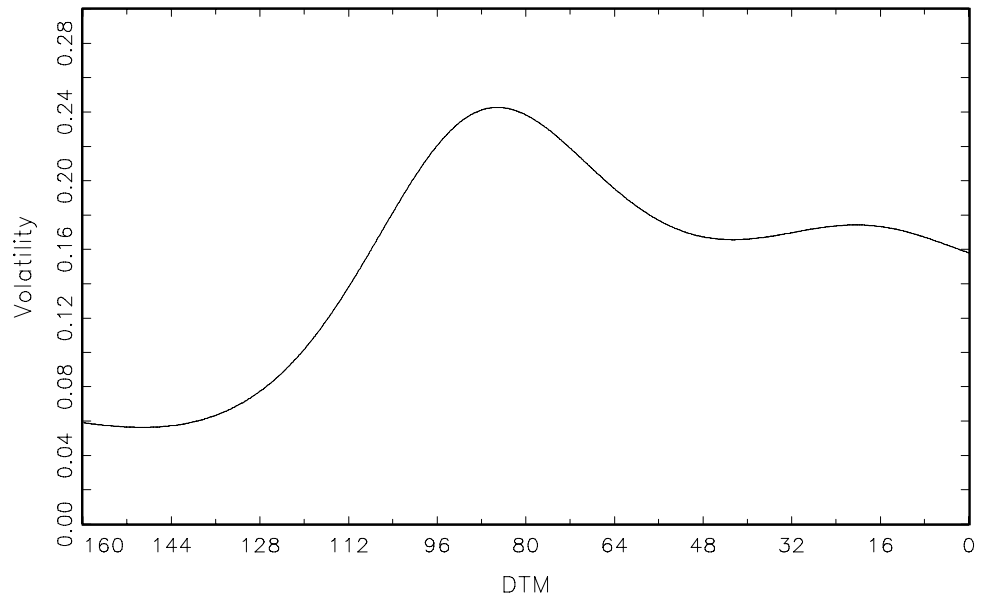

Figure 3: 20 tick Price Change Volatility vs. Time, $16 \mathrm{dtm}$ BUND Future trading, LIFFE

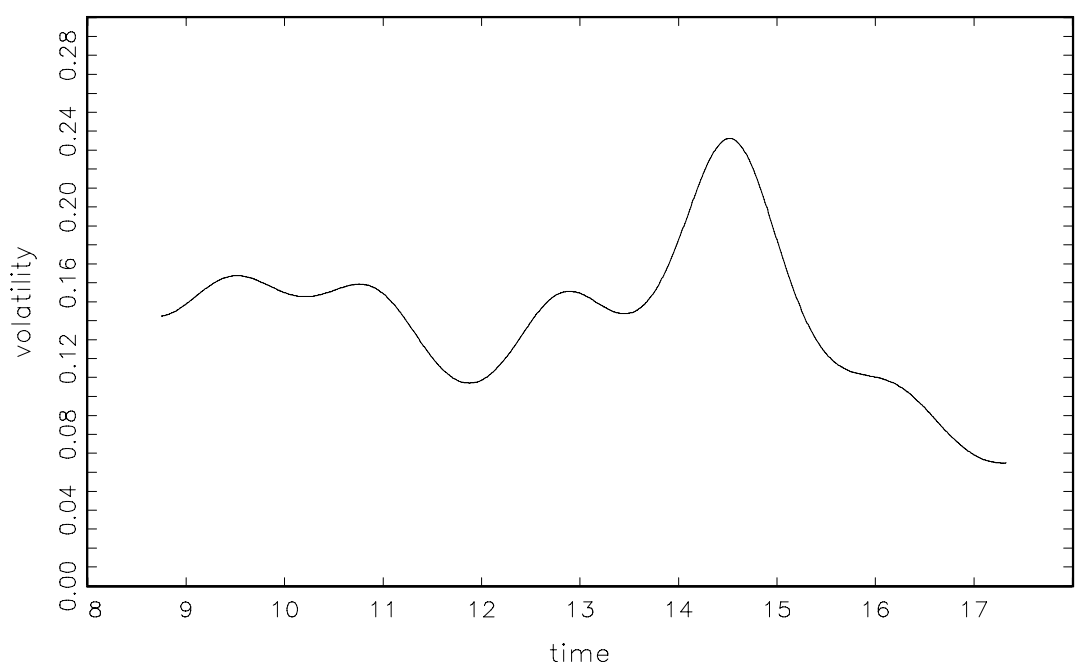


Figur 4: 20 Tick Price Change Volatility vs. DTM, 14:00, With Heterogeneity, BUND Future Trading, LIFFE

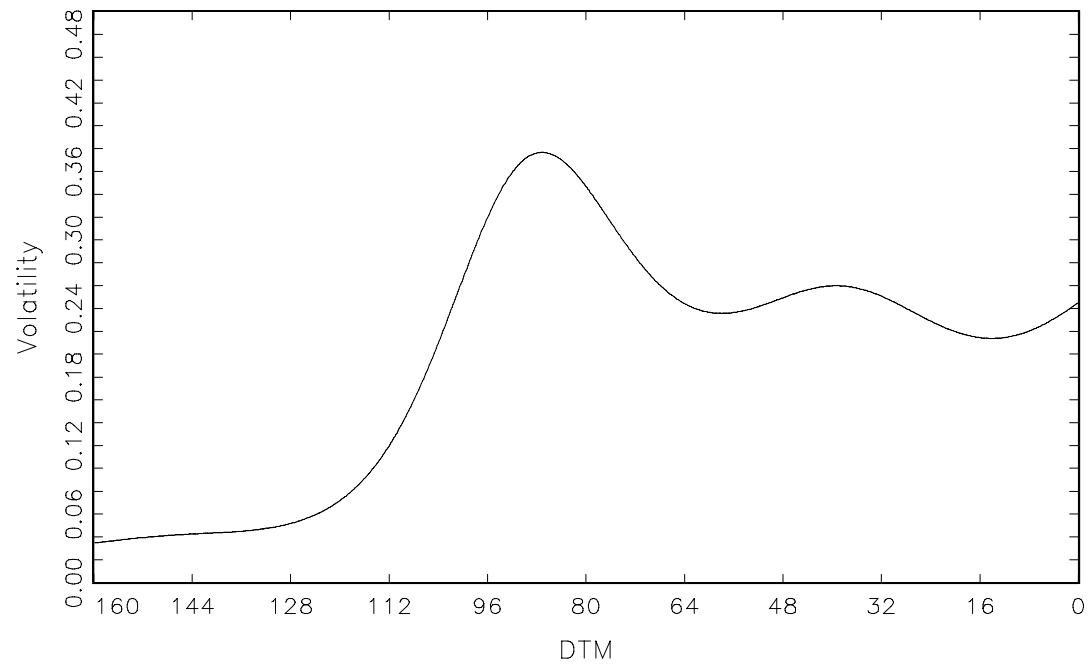

Figur 5: 20 Tick Price Change Volatility vs. Time, 16 DTM, With Heterogeneity, BUND Future Trading, LIFFE

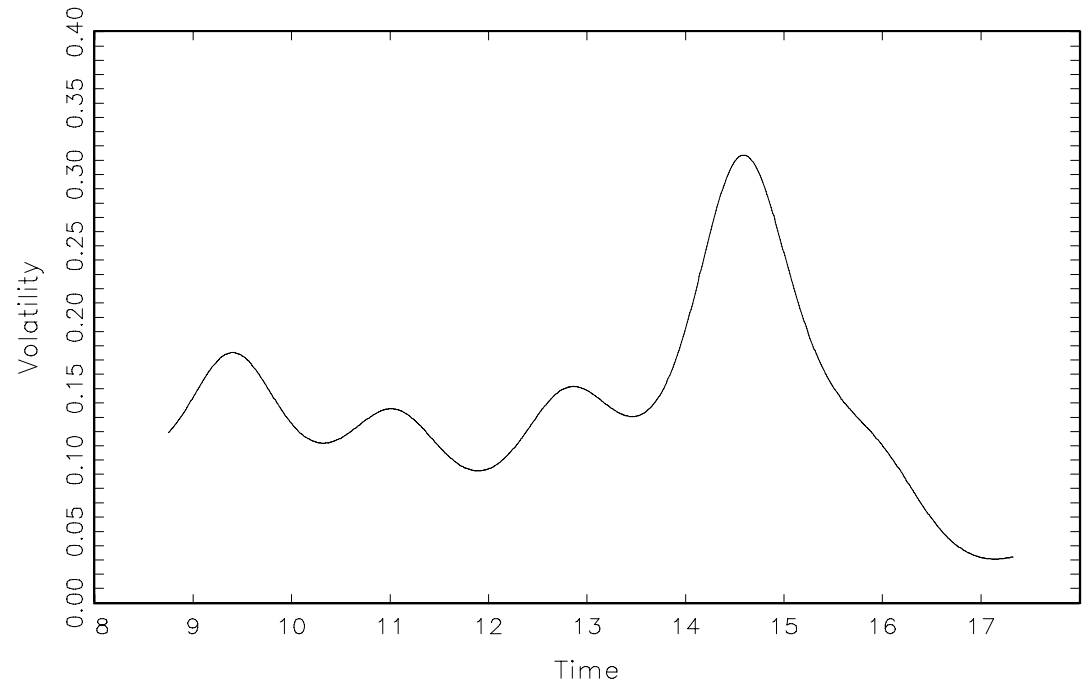

